\title{
Extending Markov Processes in Weak Duality by Poisson Point Processes of Excursions
}

\author{
Zhen-Qing Chen ${ }^{1}$, Masatoshi Fukushima ${ }^{2}$, and Jiangang Ying ${ }^{3}$ \\ 1 Department of Mathematics, University of Washington, Seattle, WA 98195, \\ USA, zchen@math.washington.edu. The research of this author is supported in \\ part by NSF Grant DMS-0303310 . \\ 2 Department of Mathematics, Kansai University, Suita, Osaka 564-8680, Japan, \\ fuku $2 @ m x 5$. canvas.ne.jp. The research of this author is supported in part by \\ Grant-in-Aid for Scientific Research of MEXT No. 15540142. \\ 3 Department of Mathematics, Fudan University, Shanghai, China, \\ jgying@fudan.edu.cn. The research of this author is supported in part by \\ NSFC No. 10271109.
}

Summary. Let $a$ be a non-isolated point of a topological space $E$. Suppose we are given standard processes $X^{0}$ and $\widehat{X}^{0}$ on $E_{0}=E \backslash\{a\}$ in weak duality with respect to a $\sigma$-finite measure $m$ on $E_{0}$ which are of no killings inside $E_{0}$ but approachable to $a$. We first show that their extensions $X$ and $\widehat{X}$ to $E$ admitting no sojourn at $a$ and keeping the weak duality are uniquely determined by the approaching probabilities of $X^{0}, \widehat{X}^{0}$ and $m$ up to a non-negative constant $\delta_{0}$ representing the killing rate of $X$ at $a$. We then construct, starting from $X^{0}$, such $X$ by piecing together returning excursions around $a$ and a possible non-returning excursion including the instant killing. This extends a recent result by M. Fukushima and H. Tanaka [16] which treats the case where $X^{0}, X$ are $m$-symmetric diffusions and $X$ admits no sojourn nor killing at $a$. Typical examples of jump type symmetric Markov processes and non-symmetric diffusions on Euclidean domains are given at the end of the paper.

Dedicated to Professor Kiyosi Itô on the occasion of his 90th birthday

\section{Introduction}

Let $a$ be a non-isolated point of a topological space $E$ and $X^{0}=\left\{X_{t}^{0}, \zeta^{0}, \mathbf{P}_{x}^{0}\right\}$ be a strong Markov process on $E_{0}=E \backslash\{a\}$ which admits no killings inside $E_{0}$ and satisfies

$$
\varphi(x):=\mathbf{P}_{x}^{0}\left(\zeta^{0}<\infty, X_{\zeta^{0}-}^{0}=a\right)>0 \quad \text { for every } x \in E_{0} .
$$

We are concerned with a strong Markovian extension $X$ of $X^{0}$ from $E_{0}$ to $E$ such that $X$ admits no sojourn at the one-point set $\{a\}$. Natural questions 
arise: is $X$ uniquely determined by $X^{0}$ and how can it be constructed from $X^{0}$ ?

When both $X^{0}$ and $X$ are required to be diffusions that are symmetric with respect to a $\sigma$-finite measure $m$ on $E_{0}$ with $m(\{a\})=0$, affirmative answers to these questions were given quite recently in M. Fukushima and H. Tanaka [16]. It is shown in [16] that the entrance law and the absorption rate for the absorbed Poisson point processes of excursions attached to $X$ away from $a$ (due to K. Itô [24] and P.A. Meyer [27]) are uniquely determined by the approaching probability $\varphi$ to $a$ for $X^{0}$ and the measure $m$, yielding the uniqueness of the extension $X$ that admits no sojourn nor killing at the point $a$. Conversely such extension $X$ can be constructed from $X^{0}$ by piecing together the associated returning excursions and possibly a non-returning one away from $a$.

The purpose of the present paper is to generalize the stated results of [16] to general standard processes $X^{0}$ and $X$ which are not necessarily symmetric but admitting weak dual standard processes $\widehat{X}^{0}$ and $\widehat{X}$, respectively. We can no longer use the Dirichlet form theory which has played an important role in $[16]$.

Nevertheless, the entrance law and the absorption rate for the absorbed Poisson point process of excursions of $X$ at the point $a$ can still be identified in $\S 2$ and $\S 3$ in terms of the approaching probabilities to $a$ by $X^{0}$ and $\widehat{X^{0}}$ and $m$ owing to the recent works on the exit system by P.J. Fitzsimmons and R.G. Getoor [12] and by the present authors [5]. It turns out that we must allow the killings of $X$ and $\widehat{X}$ at the point $a$ in order to preserve the duality of $X^{0}$ and $\widehat{X}^{0}$ so that the uniqueness of extensions holds only up to a parameter $\delta_{0}$ that represents the killing rate of $X$ at $a$ (see Theorem 4.2).

In $\S 5$, we shall construct such an extension $X$ starting from $X^{0}$ by piecing together the returning excursions around $a$ and possibly a non-returning excursion from $a$ including a killing at $a . X^{0}$ and its dual $\widehat{X^{0}}$ are assumed to be of no killings inside $E_{0}$. The sample path of the constructed process $X$ is cadlag and is continuous at the times $t$ when $X_{t}=a$. If $X^{0}$ is of continuous sample path, then so is $X$. In this construction, we can proceed along essentially the same line laid in [16] although some natural additional conditions on $X^{0}$ and $\widehat{X}^{0}$ including an off-diagonal finiteness of jumping measures will be required due to the lack of the symmetry and the path continuity. But we shall see that an integrability condition of the $\alpha$-order approaching probability being imposed on $X^{0}$ in [16] can be removed under a fairly general circumstance.

As a typical example of a jump type Markov process, we consider in $\S 6$ the case where $X^{0}$ is a censored symmetric $\alpha$-stable process on an open set of $\mathbb{R}^{n}$ studied by K. Bogdan, K. Burdzy and Z.-Q. Chen [3]. An example is also given on extending non-symmetric diffusions in Euclidean domains. Finally we remark at the end of $\S 6$ that the present results on the one point extensions can be applied to obtaining an extension to infinitely many points. 


\section{Exit system and point process of excursions around a point}

Let $E$ be a Lusin space (i.e. a space that is homeomorphic to a Borel sunset of a compact metric space), $\mathcal{B}(E)$ be the Borel $\sigma$-algebra on $E$ and $m$ be a $\sigma$-finite Borel measure on $E$. We consider a pair of Borel right processes $X=\left(X_{t}, \zeta, \mathbf{P}_{x}\right)$ and $\widehat{X}=\left(\widehat{X}_{t}, \widehat{\zeta}, \widehat{\mathbf{P}}_{x}\right)$ on $E$ that are in weak duality with respect to $m$ :

(C.1) $\int_{E} \widehat{G}_{\alpha} f(x) g(x) m(d x)=\int_{E} f(x) G_{\alpha} g(x) m(d x)$

for every $f, g \in \mathcal{B}^{+}(E)$ and $\alpha>0$, where $G_{\alpha}, \widehat{G}_{\alpha}$ denote the resolvents of $X, \widehat{X}$ respectively.

We fix a point $a \in E$ which is regular for $\{a\}$ with respect to $X$ :

(C.2) $\mathbf{P}_{a}\left(\sigma_{a}=0\right)=1$.

Here $\sigma_{a}=\inf \left\{t>0: X_{t}=a\right\}$ with the convention of inf $\varnothing:=\infty$.

Under (C.1), we may and do assume that both $X$ and $\widehat{X}$ are of cadlag paths up to their life times (c. $[21, \S 9])$.

Let $E_{0}:=E \backslash\{a\}, m_{0}:=\left.m\right|_{E_{0}}$, and

$$
\varphi(x):=\mathbf{P}_{x}\left(\sigma_{a}<\infty\right), \quad u_{\alpha}(x):=\mathbf{E}_{x}\left[e^{-\alpha \sigma_{a}}\right] \quad \text { for every } x \in E .
$$

The corresponding functions for $\widehat{X}$ will be denoted by $\widehat{\varphi}$ and $\widehat{u}_{\alpha}(x)$, respectively. For $u, v \in \mathcal{B}^{+}\left(E_{0}\right),(u, v)$ will denote the inner product of $u$ and $v$ in $L^{2}\left(E_{0} ; m_{0}\right)$, that is, $(u, v):=\int_{E_{0}} u(x) v(x) m_{0}(d x)$.

Denote by $X^{0}=\left(X_{t}^{0}, \zeta^{0}, \mathbf{P}_{x}^{0}\right)$ and $\widehat{X}^{0}=\left(\widehat{X}_{t}^{0}, \widehat{\zeta}^{0}, \widehat{\mathbf{P}}_{x}^{0}\right)$ the subprocesses of $X$ and $\widehat{X}$ killed upon leaving $E_{0}$, respectively. It is known that they are in weak duality with respect to $m_{0}$. The $X^{0}$-energy functional $L^{(0)}\left(\widehat{\varphi} \cdot m_{0}, v\right)$ of the $X^{0}$-excessive measure $\widehat{\varphi} \cdot m_{0}$ and an $X^{0}$-excessive function $v$ is then well defined by

$$
L^{(0)}\left(\widehat{\varphi} \cdot m_{0}, v\right)=\lim _{t \downarrow 0} \frac{1}{t}\left(\widehat{\varphi}-\widehat{P}_{t}^{0} \widehat{\varphi}, v\right),
$$

where $\widehat{P}_{t}^{0}$ is the transition semigroup of $\widehat{X}^{0}$ (see [16, Lemma 2.1]).

We shall now work with the exit system of $X$ for the point $a$. To this end, it is convenient to take as the sample space $\Omega$ of the process $X$ the space of all paths $\omega$ on $E_{\Delta}=E \cup \Delta$ which are cadlag up to the life time $\zeta(\omega)$ and stay at the cemetery $\Delta$ after $\zeta$. Thus, $X_{t}(\omega)$ is just $t$-th coordinate of $\omega . \Omega$ is equipped with the minimal completed admissible filtration $\left\{\mathcal{F}_{t}, t \geq 0\right\}$ for $\left\{X_{t}, t \geq 0\right\}$. The shift operator $\theta_{t}$ is defined by $X_{s}\left(\theta_{t} \omega\right)=X_{s+t}(\omega), s \geq 0$. We also introduce an operator $k_{t}, t \geq 0$, on $\Omega$ defined by

$$
X_{s}\left(k_{t} \omega\right)=\left\{\begin{array}{lll}
X_{s}(\omega) & \text { if } \quad s<t \\
\Delta & \text { if } \quad s \geq t
\end{array}\right.
$$


We adopt the usual convention that any numerical function of $E$ is extended to $E_{\Delta}$ by setting its value at $\Delta$ to be zero.

Let us consider the random time set $M(\omega)$

$$
M(\omega):=\overline{\left\{t \in[0, \infty): X_{t}(\omega)=a\right\}},
$$

where ${ }^{-}$indicates the closure in $[0, \infty)$. The random set $M(\omega)$ is closed and homogeneous on $[0, \infty)$.

Define $R_{t}(\omega):=t+\sigma_{a}\left(\theta_{t} \omega\right)$ for every $t>0$ and $L(\omega):=\sup \{s>0: s \in$ $M(\omega)\}$, with the convention that $\sup \emptyset:=0$. The connected components of the open set $[0, \infty) \backslash M(\omega)$ are called the excursion intervals. The collection of the strictly positive left end points of excursion intervals will be denoted by $G(\omega)$. We can easily see that

$$
t \in G(\omega) \quad \text { if and only if } \quad R_{t-}(\omega)<R_{t}(\omega),
$$

and in this case $R_{t-}(\omega)=t$. In particular, $L(\omega) \in G(\omega)$ whenever $L(\omega)<\infty$. We further define the operator $i_{t}, t \geq 0$, on $\Omega$ by $i_{t}=k_{\sigma_{a}} \circ \theta_{t}$. Then

$$
\left\{i_{s} \omega: s \in G\right\} \quad \text { and } \quad\left\{i_{s} \omega: s \in G, R_{s}<\infty\right\}
$$

are by definition the collection of excursions and the collection of returning excursions respectively of the path $\omega$ away from $F$, while $i_{L(\omega)}(\omega)=\theta_{L(\omega)}(\omega)$ is the non-returning excursion whenever $L(\omega)<\infty$.

Note that those excursions belong to the excursion space $W$ specified by

$$
W=\left\{k_{\sigma_{a}} \omega: \omega \in \Omega, \sigma_{a}(\omega)>0\right\},
$$

which can be decomposed as

$$
W=W^{+} \cup W^{-} \cup\{\partial\}
$$

with

$$
W^{+}=\left\{w \in W: \sigma_{a}<\infty\right\} \quad \text { and } \quad W^{-}=\left\{w \in W: \sigma_{a}=\infty \text { and } \zeta>0\right\} .
$$

Here $\partial$ denotes the path identically equal to $\Delta$.

The unit mass $\delta_{\{a\}}$ concentrated at the point $a$ is smooth in the sense of [11] because $\{a\}$ is not semipolar by the assumption (C.2). Hence there is a unique positive continuous additive functional (PCAF in abbreviation) $\ell=\left\{\ell_{t}, t \geq 0\right\}$ of $X$ with Revuz measure $\delta_{\{a\}}$. Clearly $\ell$ is supported by $\{a\}$ and any PCAF of $X$ supported by $\{a\}$ is a constant multiple of $\ell$. We call $\ell$ the local time of $X$ at the point $a$.

Since the point $a$ is assumed to be regular for $\{a\},\left\{t \geq 0: X_{t}=a\right\}$ has no isolated points, and the equilibrium 1-potential $\mathbf{E}_{x}\left[e^{-\sigma_{a}}\right]$ is regular in the sense of [2, Definition IV.3.2] because $\mathbf{E}^{x}\left[e^{-\sigma_{a}}\right]=c \mathbf{E}_{x}\left[\int_{0}^{\infty} e^{-t} d \ell_{t}\right]$ on $E$ for some $c>0$. Thus according to [26, §9] (see also [1], [8], [12] and [20])), there exists a unique $\sigma$-finite measure $\mathbf{P}^{*}$ on $\Omega$ carried by $\left\{\sigma_{a}>0\right\}$ and satisfying 


$$
\mathbf{P}^{*}\left[1-e^{-\sigma_{a}}\right]<\infty
$$

such that

$$
\mathbf{E}_{x}\left[\sum_{s \in G} Z_{s} \cdot \Gamma \circ \theta_{s}\right]=\mathbf{P}^{*}(\Gamma) \cdot \mathbf{E}_{x}\left[\int_{0}^{\infty} Z_{s} d \ell_{s}\right] \quad \text { for } x \in E
$$

for every non-negative predictable process $Z$ and every non-negative random variable $\Gamma$ on $\Omega$. Here $\mathbf{E}^{*}$ is the expectation under the law $\mathbf{P}^{*}$. The pair $\left(\mathbf{P}^{*}, \ell\right)$ is the predictable version of the exist system for $a$ originated in Maisonneuve $[26, \S 9]$. The measure $\mathbf{P}^{*}$ is Markovian with respect to the transition semigroup of $X$. We are particularly concerned with the $\sigma$-finite measure $\mathbf{Q}^{*}$ on the space of excursions $W$ induced from $\mathbf{P}^{*}$ by $\mathbf{Q}^{*}(\Gamma)=\mathbf{E}_{*}\left(\Gamma \circ k_{\sigma_{a}}\right)$. The measure $\mathbf{Q}^{*}$ is Markovian with respect to the semigroup $\left\{P_{t}^{0}, t \geq 0\right\}$ of $X^{0}$ and satisfies

$$
\mathbf{E}_{x}\left[\sum_{s \in G} Z_{s} \cdot \Gamma \circ i_{s}\right]=\mathbf{Q}^{*}[\Gamma] \cdot \mathbf{E}_{x}\left[\int_{0}^{\infty} Z_{s} d \ell_{s}\right], \quad x \in E,
$$

for every non-negative predictable process $Z_{s}$ and every non-negative random variable $\Gamma$ on $W$.

We define for $f \in \mathcal{B}^{+}(E)$

$$
\nu_{t}(f):=\mathbf{Q}^{*}\left[f\left(X_{t}\right)\right]=\mathbf{E}^{*}\left[f\left(X_{t}\right) ; t<\sigma_{a}\right], \quad t>0 .
$$

By the Markov property of $\mathbf{Q}^{*}$, we readily see that $\left\{\nu_{t}: t>0\right\}$ is an entrance law for $X^{0}: \nu_{t} P_{s}^{0}=\nu_{t+s}$.

Proposition 2.1 (i) $\left\{\nu_{t}\right\}_{t>0}$ is the unique $X^{0}$-entrance law characterized by

$$
\widehat{\varphi} \cdot m_{0}=\int_{0}^{\infty} \nu_{t} d t
$$

Moreover $\nu_{t}\left(E_{0}\right)$ is finite for each $t>0$.

(ii) $\mathbf{Q}^{*}\left[W^{-}\right]=L^{(0)}\left(\widehat{\varphi} \cdot m_{0}, 1-\varphi\right)$.

Proof. (i). We put $\check{\nu}_{\alpha}(f)=\int_{0}^{\infty} e^{-\alpha t} \nu_{t}(f) d t$. Then, for $f \in \mathcal{B}_{b}^{+}(E)$ and for $v \in C_{b}(E)$ vanishing at $a$, we have, using (C.1), (2.6) and the Revuz formula $[21,(2.13)]$

$$
\begin{aligned}
& \left(\widehat{u}_{\alpha}, v\right) \widehat{G}_{\alpha} f(a)=\left(\widehat{G}_{\alpha} f-\widehat{G}_{\alpha}^{0} f, v\right)=\left(f, G_{\alpha} v-G_{\alpha}^{0} v\right) \\
= & \mathbf{E}_{f \cdot m}\left[\int_{\sigma_{a}}^{\infty} e^{-\alpha t} v\left(X_{t}\right) 1_{M^{c}}(t) d t\right]=\mathbf{E}_{f \cdot m}\left[\sum_{s \in M} \int_{s}^{s+\sigma_{a} \circ \theta_{s}} e^{-\alpha t} v\left(X_{t}\right) d t\right] \\
= & \mathbf{E}_{f \cdot m}\left[\sum_{s \in M} e^{-\alpha s} \int_{0}^{\sigma_{a}} e^{-\alpha t} v\left(X_{t}\right) d t \circ \theta_{s}\right]=\check{\nu}_{\alpha}(v) \mathbf{E}_{f \cdot m}\left[\int_{0}^{\infty} e^{-\alpha s} d \ell_{s}\right] \\
= & \check{\nu}_{\alpha}(v) \widehat{G}_{\alpha} f(a) .
\end{aligned}
$$


Hence

$$
\widehat{u}_{\alpha} \cdot m_{0}=\check{\nu}_{\alpha}
$$

from which (2.7) follows by letting $\alpha \downarrow 0$. Since $\widehat{\varphi} \cdot m_{0}$ is a purely excessive measure of $X^{0}$, the uniqueness follows (cf. [20]). The finiteness of $\nu_{t}$ follows from (2.4).

(ii). By (i) and [5, Lemma 3.1], $L^{(0)}\left(\widehat{\varphi} \cdot m_{0}, v\right)=\lim _{t \downarrow 0} \nu_{t}(v)$ for any $X^{0}$-excessive function $v$. Hence

$$
\begin{aligned}
L^{(0)}\left(\widehat{\varphi} \cdot m_{0}, 1-\varphi\right) & =\lim _{t \downarrow 0} \mathbf{Q}^{*}\left[(1-\varphi)\left(X_{t}\right)\right]=\lim _{t \downarrow 0} \mathbf{Q}^{*}\left[1_{\sigma_{a}=\infty} \circ \theta_{t} ; t<\zeta \wedge \sigma_{a}\right] \\
& =\mathbf{Q}^{*}\left[W^{-}\right] .
\end{aligned}
$$

Remark 1. In the next section, we shall identify $\mathbf{Q}^{*}$ with the characteristic measure $\mathbf{n}$ of the absorbed Poisson point process of excursions associated with $\ell$. Proposition 2.1 was first proved by Fukushima-Tanaka [16] for $\mathbf{n}$ in the case that $X$ is an $m$-symmetric diffusion by making use of the Dirichlet form of $X$. In a recent paper of Fitzsimmons-Getoor [12], various properties of some basic quantities for the exit system of a one point set including those in the above proposition have been obtained in the most general setting that $X$ is just a Borel right process with an excessive measure $m$, in which case $\widehat{X}$ can be taken to be a dual left continuous moderate Markov process. But the present proof, taken from a recent paper by Chen-Fukushima-Ying [5], is simpler under the condition (C.1) as far as Proposition 2.1 is concerned.

The next proposition is taken from Fitzsimmons-Getoor [12, (2.10) and (2.17)]. Recall that $L(\omega):=\sup M(\omega)$.

Proposition 2.2 Put $\delta=\mathbf{P}^{*}\left(\sigma_{a}=\infty\right)$. Then the followings are true:

(i) $\mathbf{P}_{a}\left(\ell_{\infty}>t\right)=\exp (-\delta t), \quad t>0$.

(ii) $\mathbf{P}_{a}(L<\infty)=0$ or 1 according to $\delta=0$ or $\delta>0$.

Let $\left\{\tau_{t}, t \geq 0\right\}$ be the right continuous inverse of $\ell=\left\{\ell_{t}, t \geq 0\right\}$, that is,

$$
\tau_{t}:=\inf \left\{s \geq 0: \ell_{s}>t\right\},
$$

with the convention that $\inf \emptyset=\infty$. Since $\ell$ is supported by $a$, we have (cf. $[4, \S 5]) \mathbf{P}_{a}$-a.s.

$$
\tau_{\ell_{t}}=R_{t} \quad \text { for every } t \geq 0 .
$$

We see from the above that, after removing from $\Omega$ a $\mathbf{P}_{a}$-negligible set,

$$
L(\omega)<\infty \text { if and only if } \ell_{\infty}(\omega)<\infty,
$$

and in this case, 


$$
\ell_{\infty}(\omega)=\ell_{L}(\omega), \quad \tau_{\ell_{\infty}-}(\omega)=L(\omega) \quad \text { and } \quad \tau_{\ell_{\infty}}(\omega)=\infty .
$$

Hence, if we let

$$
J_{\ell}(\omega):=\left\{s \in(0, \infty): \tau_{s-}(\omega)<\tau_{s}(\omega)\right\}
$$

then

$$
J_{\ell}(\omega):=\left\{\ell_{t}: t \in G(\omega)\right\}
$$

and $s \in J_{\ell}(\omega)$ implies that $s=\ell_{t}(\omega)$ for some $t \in G(\omega)$ with $\tau_{s-}(\omega)=$ $R_{t-}(\omega)=t$ and $\tau_{s}(\omega)=R_{t}(\omega)$.

In particular, $\ell_{\infty}(\omega) \in J_{\ell}(\omega)$ whenever it is finite.

Finally the $W$-valued point process $\mathbf{p}=\mathbf{p}(\omega)$ associated with the local time $\ell$ is introduced by

$$
\mathcal{D}_{\mathbf{p}(\omega)}=J_{\ell}(\omega) \quad \text { and } \quad \mathbf{p}_{s}(\omega)=i_{\tau_{s-}} \omega \text { for } s \in \mathcal{D}_{\mathbf{p}(\omega)} .
$$

Note that $\left\{\mathbf{p}_{s}(\omega): s \in \mathcal{D}_{\mathbf{p}(\omega)}\right\} \subset W$ and $\left\{\mathbf{p}_{s}(\omega): s \in \mathcal{D}_{\mathbf{p}(\omega)}, \tau_{s}<\infty\right\} \subset W^{+}$ is the collections of excursions and of the returning excursions away from $a$, respectively, while $\mathbf{p}_{\ell_{\infty}}(\omega)\left(=\theta_{L}(\omega)\right) \in W^{-} \cup\{\partial\}$ is the non-returning excursion whenever $\ell_{\infty}(\omega)<\infty$ or, equivalently, $L(\omega)<\infty$.

The counting measure of $\mathbf{p}$ is defined by

$$
n_{\mathbf{p}}((s, t], \Lambda)=\sum_{u \in \mathcal{D}_{\mathbf{p}} \cap(s, t]} 1_{\Lambda}\left(\mathbf{p}_{u}\right), \quad \Lambda \in \mathcal{B}(W),
$$

and $n_{\mathbf{p}}(t, \Lambda)=n_{\mathbf{p}}((0, t], \Lambda)$ is then $\mathcal{F}_{\tau_{t}}$-adapted as a process in $t \geq 0$.

Using (2.10), we now make the time substitute in the relation (2.6) to obtain

$$
\mathbf{E}_{a}\left[\sum_{s \in J_{\ell}} Z_{\tau_{s-}} \cdot \Gamma \circ i_{\tau_{s-}}\right]=\mathbf{Q}^{*}[\Gamma] \cdot \mathbf{E}_{a}\left[\int_{0}^{\ell_{\infty}} Z_{\tau_{s}} d s\right] .
$$

Inserting the predictable process $Z_{u}=1_{\left(0, \tau_{t-}\right]}(u)$, we arrive at the formula holding for the counting measure of the point process $\mathbf{p}$ associated with $\ell$ :

$$
\mathbf{E}_{a}\left[n_{\mathbf{p}}(t, \Lambda)\right]=\mathbf{Q}^{*}[\Lambda] \cdot \mathbf{E}_{a}\left[t \wedge \ell_{\infty}\right] \quad \text { for every } t \geq 0 \text { and } \Lambda \in \mathcal{B}(W) .
$$

This formula will be utilized in the next section.

\section{Characteristic measure of absorbed Poisson point process}

In this section, we continue to work with the setting in $\S 2$ and investigate properties of the point process $\left(\mathbf{p}_{s}, \mathcal{D}_{\mathbf{p}}\right)$ defined by $(2.11)$ for the local time 
$\ell=\left\{\ell_{t}, t \geq 0\right\}$ at the point $a$. By the observation made after (2.11), it then holds that

$$
\ell_{\infty}=T \text { where } T=\inf \left\{s>0: \mathbf{p}_{s} \in W^{-} \cup\{\partial\}\right\} .
$$

In view of Proposition 2.2, $T$ is exponentially distributed with parameter $\delta=\mathbf{P}^{*}\left(\sigma_{a}=\infty\right)$.

Lemma 3.1 Under measure $\mathbf{P}_{a}, \mathbf{p}$ is an absorbed Poisson point process with absorption time $T$ in Meyer's sense ([27]), that is,

$$
\begin{aligned}
& \mathbf{P}_{a}\left(n_{\mathbf{p}}\left(\left(r+s_{1}, r+t_{1}\right], \Lambda_{1}\right) \in H_{1}, \cdots, n_{\mathbf{p}}\left(\left(r+s_{n}, r+t_{n}\right], \Lambda_{n}\right) \in H_{n} \mid \mathcal{F}_{\tau_{r}}\right) \\
= & 1_{\{T>r\}} \mathbf{P}_{a}\left(n_{\mathbf{p}}\left(\left(s_{1}, t_{1}\right], \Lambda_{1}\right) \in H_{1}, \cdots, n_{\mathbf{p}}\left(\left(s_{n}, t_{n}\right], \Lambda_{n}\right) \in H_{n}\right) \\
& +1_{\{T \leq r\}} 1_{H_{1}}(0) \cdots 1_{H_{n}}(0),
\end{aligned}
$$

for any $s_{1}<t_{1}, \cdots, s_{n}<t_{n}, H_{1}, \cdots, H_{n} \subset \mathbb{Z}_{+}, r>0, \Lambda_{1}, \cdots, \Lambda_{n} \in \mathcal{B}(W)$.

Proof. The proof is the same as in [27, §2] although [27] considered only the conservative case. In fact, the identity $\tau_{r+u}=\tau_{r}+\tau_{u} \circ \theta_{\tau_{r}}$ implies $n_{\mathbf{p}}((r+$ $s, r+t], \Lambda)=n_{\mathbf{p}}((s, t], \Lambda) \circ \theta_{\tau_{r}}$ and consequently we see from (3.1) and the strong Markov property of $X$ that the left hand side of (3.2) (with $n=1$ ) equals

$$
\begin{aligned}
\mathbf{P}_{X_{\tau_{r}}}\left(n_{\mathbf{p}}((s, t], \Lambda) \in H\right)=1_{\{T>r\}} \mathbf{P}_{a}\left(n_{\mathbf{p}}((s, t], \Lambda) \in H\right) \\
+1_{\{T \leq r\}} \mathbf{P}_{\Delta}\left(n_{\mathbf{p}}((s, t], \Lambda) \in H\right),
\end{aligned}
$$

whose last factor is equal to $1_{H}(0)$.

By virtue of $[27, \S 1]$, there is on a certain probability space $(\widetilde{\Omega}, \widetilde{\mathbf{P}})$ a $W$ valued Poisson point process $\widetilde{\mathbf{p}}=\{\widetilde{\mathbf{p}}, s>0\}$ with domain $\mathcal{D}_{\widetilde{\mathbf{p}}}$ satisfying the following property.

Let $\widetilde{T}=\inf \left\{s>0: \widetilde{\mathbf{p}}_{s} \in W^{-} \cup\{\partial\}\right\}$ and consider the stopped process $\left\{\overline{\mathbf{p}}_{s}, s>0\right\}$ :

$$
\overline{\mathbf{p}}_{s}=\widetilde{\mathbf{p}}_{s} \quad \text { for } \quad s \in \mathcal{D}_{\overline{\mathbf{p}}}=\mathcal{D}_{\widetilde{\mathbf{p}}} \cap(0, \widetilde{T}] .
$$

Then the point process $\left\{\mathbf{p}_{s}, s>0\right\}$ under $\mathbf{P}_{a}$ and $\left\{\overline{\mathbf{p}}_{s}, s>0\right\}$ under $\widetilde{\mathbf{P}}$ are equivalent in law.

Let us denote by $\mathbf{n}$ the characteristic measure of the $W$-valued Poisson point process $\{\widetilde{\mathbf{p}}, s>0\}$.

Theorem 3.2 It holds that

$$
\mathbf{n}=\mathbf{Q}^{*}
$$

Therefore $\mathbf{n}$ is a $\sigma$-finite measure on $W$ with $\mathbf{n}\left(\sigma_{a}>t\right)<\infty$ for every $t>0$, and $\mathbf{n}$ is Markovian with respect to the transition semigroup $\left\{P_{t}^{0}, t \geq 0\right\}$ of $X^{0}$. The $X^{0}$-entrance law $\left\{\nu_{t}, t>0\right\}$ of $\mathbf{n}$ defined by 


$$
\nu_{t}(f)=\mathbf{n}\left(f\left(X_{t}\right) ; t<\sigma_{a}\right), \quad t>0, \quad f \in \mathcal{B}^{+}(E)
$$

is characterized by

$$
\int_{0}^{\infty} \nu_{t} d t=\widehat{\varphi} \cdot m_{0}
$$

Define $\delta_{0}$ by

$$
\delta_{0}=\mathbf{n}(\zeta=0) .
$$

Then $\widetilde{T}$ is exponentially distributed with parameter $L^{(0)}\left(\widehat{\varphi} \cdot m_{0}, 1-\varphi\right)+\delta_{0}$ :

$$
\widetilde{\mathbf{P}}(\widetilde{T}>t)=\exp \left(-t\left(L^{(0)}\left(\widehat{\varphi} \cdot m_{0}, 1-\varphi\right)+\delta_{0}\right)\right) \quad \text { for every } t>0 .
$$

Moreover, $\nu_{t}\left(E_{0}\right)<\infty$ for each $t>0$ and $L^{(0)}\left(\widehat{\varphi} \cdot m_{0}, 1-\varphi\right)<\infty$.

Proof. Since $\left\{\widetilde{\mathbf{p}}_{s}: s \in \mathcal{D}_{\widetilde{\mathbf{p}}}, \widetilde{\mathbf{p}}_{s} \in W^{+}\right\}$and $\widetilde{T}$ are independent, we have by

$\mathbf{E}_{a}\left[n_{\mathbf{p}}(t, \Lambda)\right]=\widetilde{\mathbf{E}}\left[\sum_{u \in \mathcal{D}_{\widetilde{\mathbf{p}}} \cap(0, t \wedge \widetilde{T}]} 1_{\Lambda}\left(\widetilde{\mathbf{p}}_{u}\right)\right]=\mathbf{n}(\Lambda) \cdot \widetilde{\mathbf{E}}[t \wedge \widetilde{T}]=\mathbf{n}(\Lambda) \cdot \mathbf{E}_{a}\left[t \wedge \ell_{\infty}\right]$,

which compared with (2.14) leads us to (3.4).

Identities (3.5) and (3.7) are the consequences of Propositions 2.1 as

$$
\mathbf{Q}^{*}\left(W^{-} \cup\{\partial\}\right)=\mathbf{Q}^{*}\left(W^{-}\right)+\mathbf{Q}^{*}(\{\partial\})=L^{(0)}\left(\widehat{\varphi} \cdot m_{0}, 1-\varphi\right)+\delta_{0} .
$$

Then $\sigma$-finiteness of $\mathbf{n}$ and the last statement follow from (2.4).

\section{Duality preserving one-point extension}

Let $E$ be a locally compact separable metric space, $a$ be a non-isolated point of $E$ and $m$ be a $\sigma$-finite measure on $E_{0}:=E \backslash\{a\}$. Contrarily to the preceding two sections, we shall start in this section with two given strong Markov processes $X^{0}$ and $\widehat{X}^{0}$ on $E_{0}$ that are in weak duality with respect to $m_{0}$ and have no killings inside $E_{0}$. We are concerned with their possible duality preserving extensions $X$ and $\widehat{X}$ to $E$ that admit no sojourn at $a$. It turns out that we need to allow $X$ and $\widehat{X}$ have killings at $a$ in order to guarantee their weak duality but they are unique up to a parameter $\delta_{0}$ that represents the killing rate of $X$ at $a$.

We shall assume that we are given two Borel standard processes $X^{0}=$ $\left(X_{t}^{0}, \mathbf{P}_{x}^{0}, \zeta^{0}\right)$ and $\widehat{X}^{0}=\left(\widehat{X}_{t}^{0}, \widehat{\mathbf{P}}_{x}^{0}, \widehat{\zeta}^{0}\right)$ on $E_{0}$ satisfying the next three conditions.

(A.1) $X^{0}$ and $\widehat{X}^{0}$ are in weak duality with respect to $m_{0}$; that is, for every $\alpha>0$ and $f, g \in \mathcal{B}^{+}\left(E_{0}\right)$, 


$$
\int_{E_{0}} \widehat{G}_{\alpha}^{0} f(x) g(x) m_{0}(d x)=\int_{E_{0}} f(x) G_{\alpha}^{0} g(x) m_{0}(d x),
$$

where $G_{\alpha}^{0}$ and $\widehat{G}_{\alpha}^{0}$ are the resolvent of $X^{0}$ and $\widehat{X}^{0}$, respectively.

(A.2) $X^{0}$ and $\widehat{X}^{0}$ are approachable to $\{a\}$ but admit no killings inside $E_{0}$ : for every $x \in E_{0}$,

$$
\begin{aligned}
& \mathbf{P}_{x}^{0}\left(\zeta^{0}<\infty, X_{\zeta^{0}-}^{0}=a\right)>0 \text { and } \mathbf{P}_{x}^{0}\left(\zeta^{0}<\infty, X_{\zeta^{0}-}^{0} \in E_{0}\right)=0 \\
& \widehat{\mathbf{P}}_{x}^{0}\left(\widehat{\zeta}^{0}<\infty, \widehat{X}_{\widehat{\zeta}^{0}-}^{0}=a\right)>0 \text { and } \widehat{\mathbf{P}}_{x}^{0}\left(\widehat{\zeta}^{0}<\infty, \widehat{X}_{\widehat{\zeta}^{0}-}^{0} \in E_{0}\right)=0
\end{aligned}
$$

Here for a Borel set $B \subset E$, the notation " $X_{\zeta^{0}-}^{0} \in B$ " means that the left limit of $X_{t}^{0}$ at $t=\zeta^{0}$ exists under the topology of $E$ and takes values in $B \subset E$. We use the same convention for $\widehat{X}$.

We shall use the same notations as in [16]: for $x \in E_{0}$ and $\alpha>0$,

$$
\varphi(x):=\mathbf{P}_{x}^{0}\left(\zeta^{0}<\infty, X_{\zeta^{0}-}^{0}=a\right) \text { and } u_{\alpha}(x):=\mathbf{E}_{x}^{0}\left[e^{-\alpha \zeta^{0}}: X_{\zeta^{0}-}^{0}=a\right] .
$$

As in $\S 2$, the $X^{0}$-energy functional of $X^{0}$-excessive measure $\mu$ and $X^{0}$ excessive function $v$ is denoted by $L^{(0)}(\mu, v)$. The corresponding notations for $\widehat{X}^{0}$ will be designated by $\widehat{\varphi}, \widehat{u}_{\alpha}, \widehat{L}^{(0)}$. We use $(u, v)$ to denote the inner product between $u$ and $v$ in $L^{2}\left(E_{0}, m_{0}\right)$, that is, $(u, v)=\int_{E_{0}} u(x) v(x) m_{0}(d x)$.

We say that a strong Markov process $X$ (resp. $\widehat{X}$ ) on $E$ is an extension of $X^{0}\left(\right.$ resp. $\left.\widehat{X}^{0}\right)$ if the subprocess on $E_{0}$ of $X$ (resp. $\widehat{X}$ ) killed upon hitting the point $a$ is identical in law to $X^{0}\left(\right.$ resp. $\left.\widehat{X}^{0}\right)$.

Let us now consider two Borel right processes $X=\left(X_{t}, \mathbf{P}_{x}, \zeta\right)$ and $\widehat{X}=$ $\left(\widehat{X}_{t}, \widehat{\mathbf{P}}_{x}, \widehat{\zeta}\right)$ on $E$ satisfying the next four conditions.

(1) $X$ and $\widehat{X}$ are in weak duality with respect to a $\sigma$-finite measure $m$ on $E$ with $\left.m\right|_{E_{0}}=m_{0}$.

(2) $X$ and $\widehat{X}$ are extensions of $X^{0}$ and $\widehat{X}^{0}$ respectively.

(3) The point $a$ is regular for itself with respect to $X$ :

$$
\mathbf{P}_{a}\left(\sigma_{a}=0\right)=1
$$

where $\sigma_{a}=\inf \left\{t>0: X_{t}=a\right\}$ is the hitting time of $a$ by $X$.

(4) $X$ admits no sojourn at the point $a$, that is,

$$
\mathbf{P}_{x}\left(\int_{0}^{\infty} 1_{\{a\}}\left(X_{s}\right) d s=0\right)=1 \quad \text { for every } x \in E .
$$

Under (1), we can and do assume that both $X$ and $\widehat{X}$ possess cadlag paths up to their life times. 
Proposition 4.1 Assume that the above conditions (1), (2), (3) and (4) hold. Then

(i) The measure $m$ does not charging on $\{a\}: m(\{a\})=0$

(ii) $X$ admits no jumping from $E_{0}$ to the point a: for every $x \in E_{0}$,

$$
\mathbf{P}_{x}\left(X_{t-} \in E_{0}, X_{t}=a \text { for some } t \in(0, \zeta)\right)=0,
$$

(iii) $X$ admits no jump from the point a to $E_{0}$ in the following sense:

$$
\mathbf{P}_{x}\left(X_{t-}=a, X_{t} \in E_{0} \text { for some } t \in(0, \zeta)\right)=0 \text { for q.e. } x \in E .
$$

Here q.e. means except on an m-polar set for $X$.

(iv) The one point set $\{a\}$ is not $m$-polar for $X$. Let functions $\varphi$ and $u_{\alpha}$ be defined as in (4.3). Then

$$
\varphi(x)=\mathbf{P}_{x}\left(\sigma_{a}<\infty\right) \text { and } u_{\alpha}(x)=\mathbf{E}_{x}\left[e^{-\alpha \sigma_{a}}\right] \quad \text { for } x \in E_{0} .
$$

(v) $u_{\alpha}, \widehat{u}_{\alpha} \in L^{1}\left(E_{0}, m_{0}\right)$ for every $\alpha>0$.

Proof. (i). This is immediate from (1), (4) for $X$ as

$$
\widehat{G}_{\alpha} f(a) m(\{a\})=\int_{E} f(x) G_{\alpha} 1_{\{a\}}(x) m(d x)=0 \quad \text { for every } f \in \mathcal{B}^{+}(E) .
$$

(ii). It follows from (4.1) and (2) that

$$
\mathbf{P}_{x}\left(X_{\sigma_{a}-} \in E_{0}, \sigma_{a}<\infty\right)=0 \quad \text { for every } x \in E_{0} .
$$

For any open set $O$ that has a positive distance from $\{a\}$, let $\left\{\sigma_{a}^{n}, n \geq\right.$ $0\},\left\{\eta^{n}, n \geq 0\right\}$ be the stopping times defined by

$$
\eta^{0}=0, \sigma_{a}^{0}=\sigma_{a}, \eta^{n}=\sigma_{a}^{n-1}+\sigma_{O} \circ \theta_{\sigma_{a}^{n-1}}, \sigma_{a}^{n}=\eta^{n}+\sigma_{a} \circ \theta_{\eta^{n}}
$$

with an obvious modification after one of them becomes infinity. Clearly the time set

$$
\left\{t \in(0, \zeta(\omega)): X_{t-}(\omega) \in O, X_{t}(\omega)=a\right\} \subset\left\{\sigma_{a}^{n}(\omega) ; n=0,1,2, \cdots\right\} .
$$

Thus it follows from the strong Markov property of $X$ and (4.7) that for every $x \in E_{0}$,

$$
\left.\mathbf{P}_{x} \text { (there is some } t>0 \text { such that } X_{t-} \in O, X_{t}=a\right)=0 .
$$

Letting $O$ increase to $E_{0}$ establishes (4.4).

(iii). Clearly, property (ii) also holds for $\widehat{X}$ :

$$
\widehat{\mathbf{P}}_{x}\left(\widehat{X}_{t-} \in E_{0}, \widehat{X}_{t}=a \text { for some } t \in(0, \widehat{\zeta})\right)=0 \quad \text { for every } x \in E_{0}
$$


We combine the above with a time reversal argument based on the stationary Kuznetsov process $\left(\mathbf{P}, Z_{t}, \alpha<t<\beta\right)$ associated with $X$ and $\widehat{X}$ as was formulated in $[21, \S 10]$ : the $\sigma$-finite measure $\mathbf{P}$ on a path space $D\left((-\infty, \infty), E_{\Delta}\right)$ with a random birth time $\alpha$ and a random death time $\beta$ is stationary under the time shift of the path, and furthermore, if we put

$$
\widehat{Z}_{t}=Z_{(-t)-} \text { for } t \in \mathbb{R}, \quad \widehat{\alpha}=-\beta \quad \text { and } \quad \widehat{\beta}=-\alpha,
$$

then $\left\{Z_{t}, 0 \leq t<\beta\right\}$ (resp. $\left.\left\{\widehat{Z}_{t}, 0 \leq t<\widehat{\beta}\right\}\right)$ on $\left\{Z_{0} \in E\right\}$ (resp. $\left.\left\{\widehat{Z}_{0} \in E\right\}\right)$ is a copy of $\left\{X_{t}, 0 \leq t<\zeta\right\}$ (resp. $\left.\left\{\widehat{X}_{t}, 0 \leq t<\widehat{\zeta}\right\}\right)$ under $\mathbf{P}_{m}$ (resp. $\left.\widehat{\mathbf{P}}_{m}\right)$. We shall use the formula $(10.5)$ of $[21, \S 10]$ which express a precise meaning of this property.

Consider the set

$$
\Lambda=\left\{Z_{t-}=a \text { and } Z_{t} \in E_{0}, \text { for some } t \in(\alpha, \beta)\right\} .
$$

Then

$$
\Lambda=\left\{\widehat{Z}_{t-} \in E_{0} \text { and } \widehat{Z}_{t}=a, \text { for some } t \in(\widehat{\alpha}, \widehat{\beta})\right\},
$$

and thus $\Lambda=\bigcup_{r \in \mathbb{Q}^{+}} \Lambda_{r}$ with

$$
\Lambda_{r}=\left\{\widehat{\alpha}<r<\widehat{\beta}, \widehat{Z}_{t-} \in E_{0}, \widehat{Z}_{t}=a \text { for some } t>r\right\} .
$$

According to $(10.5)$ of $[21, \S 10], \mathbf{P}\left(\Lambda_{r}\right)$ is equal to the integral of the left hand side of (4.7) with respect to $m$ for each rational $r$. Therefore $\mathbf{P}(\Lambda)=0$.

Denote by $h(x)$ the function of $x \in E$ appearing in the left hand side of (4.5). By (10.5) of $[21, \S 10]$ again, we have

$$
\begin{aligned}
\int_{E} h(x) m(d x) & =\mathbf{P}\left(Z_{t-}=a \text { and } Z_{t} \in E_{0}, \text { for some } t \in(0, \beta), \alpha<0<\beta\right) \\
& \leq \mathbf{P}(\Lambda)=0 .
\end{aligned}
$$

Consequently, $h=0 m$-a.e. and hence q.e. on $E$ because $h$ is $X$-excessive (cf. $[5, \S 2])$.

(iv). On account of [2, p.59] (see also [21, Proposition 15.7] when $E$ is a Lusin space),

$$
\mathbf{P}_{x}\left(0<\sigma_{a}^{\prime}<\sigma_{a}\right)=0, \quad \text { where } \sigma_{a}^{\prime}=\inf \left\{t: X_{t-}=a\right\}, \quad x \in E .
$$

On the other hand, (A.2) and (2) imply for $\zeta^{0}=\sigma_{a} \wedge \zeta$ that for $x \in E_{0}$,

$$
\mathbf{P}_{x}\left(\sigma_{a}<\sigma_{a}^{\prime}\right) \leq \mathbf{P}_{x}\left(\sigma_{a}<\infty, X_{\sigma_{a}-} \neq a\right) \leq \mathbf{P}_{x}\left(\zeta^{0}<\infty, X_{\zeta^{0}-} \in E_{0}\right)=0 .
$$

Hence $\mathbf{P}_{x}\left(\sigma_{a}=\sigma_{a}^{\prime}\right)=1$ and

$$
\varphi(x)=\mathbf{P}_{x}\left(\zeta^{0}<\infty, X_{\zeta^{0}-}=a\right)=\mathbf{P}_{x}\left(\sigma_{a}<\infty\right) \text { for } x \in E_{0} .
$$


In particular,

$$
\mathbf{P}_{m}\left(\sigma_{a}<\infty\right)=\int_{E_{0}} \varphi(x) m(d x)>0
$$

by (A.2) and therefore $\{a\}$ is not $m$-polar for $X$.

(v). By the strong Markov property of $\widehat{X}$,

$$
\widehat{G}_{\alpha} f(x)=\widehat{G}_{\alpha}^{0} f(x)+\widehat{u}_{\alpha}(x) \widehat{G}_{\alpha} f(a), \quad x \in E .
$$

We can take a non-negative $m$-integrable function $f$ on $E$ such that $\widehat{G}_{\alpha} f(a)>$ 0 . Then

$$
\widehat{G}_{\alpha} f(a)\left(\widehat{u}_{\alpha}, 1\right) \leq\left(\widehat{G}_{\alpha} f, 1\right)=\left(f, G_{\alpha} 1\right) \leq \frac{1}{\alpha}(f, 1)<\infty,
$$

yielding the $m_{0}$-integrability of $\widehat{u}_{\alpha}$. Similar, we have $u_{\alpha} \in L^{1}\left(E_{0}, m_{0}\right)$.

Theorem 4.2 Assume that $X$ and $\widehat{X}$ are two Borel right processes on $E$ satisfying conditions (1), (2), (3) and (4) in this section. Let $\left\{G_{\alpha}, \alpha>0\right\}$ and $\left\{\widehat{G}_{\alpha}, \alpha>0\right\}$ denote the resolvents of $X$ and $\widehat{X}$, respectively. Then there exist constants $\delta_{0} \geq 0, \widehat{\delta}_{0} \geq 0$ such that

$$
L^{(0)}\left(\widehat{\varphi} \cdot m_{0}, 1-\varphi\right)+\delta_{0}=\widehat{L}^{(0)}\left(\varphi \cdot m_{0}, 1-\widehat{\varphi}\right)+\widehat{\delta}_{0},
$$

and for every $f \in \mathcal{B}^{+}(E)$ and $\alpha>0$,

$$
\begin{aligned}
G_{\alpha} f(a) & =\frac{\left(\widehat{u}_{\alpha}, f\right)}{\alpha\left(\widehat{u}_{\alpha}, \varphi\right)+L^{(0)}\left(\widehat{\varphi} \cdot m_{0}, 1-\varphi\right)+\delta_{0}}, \\
G_{\alpha} f(x) & =G_{\alpha}^{0} f(x)+u_{\alpha}(x) G_{\alpha} f(a) \quad \text { for } x \in E_{0}, \\
\widehat{G}_{\alpha} f(a) & =\frac{\left(u_{\alpha}, f\right)}{\alpha\left(u_{\alpha}, \widehat{\varphi}\right)+\widehat{L}^{(0)}\left(\varphi \cdot m_{0}, 1-\widehat{\varphi}\right)+\widehat{\delta}_{0}}, \\
\widehat{G}_{\alpha} f(x) & =\widehat{G}_{\alpha}^{0} f(x)+\widehat{u}_{\alpha}(x) \widehat{G}_{\alpha} f(a) \quad \text { for } x \in E_{0} .
\end{aligned}
$$

Corollary 4.3 Borel right processes $X$ and $\widehat{X}$ on $E$ satisfying conditions (1)-(4) of this section are unique in law up to a parameter $\delta_{0}$ satisfying

$$
\delta_{0} \geq \max \left\{\widehat{L}^{(0)}\left(\varphi \cdot m_{0}, 1-\widehat{\varphi}\right)-L^{(0)}\left(\widehat{\varphi} \cdot m_{0}, 1-\varphi\right), 0\right\} .
$$

Proof of Theorem 4.2. In view of conditions (1)-(4) of this section and Proposition 4.1, $X$ satisfies the conditions (C.1)-(C.2) of $\S 2$ so that Theorem 3.2 is applicable to $X$.

The identity (4.12) is a simple consequence of the strong Markov property of $X$ applied to the hitting time $\sigma_{a}$. In order to show (4.11), we consider the local time $\ell=\left\{\ell_{t}, t \geq 0\right\}$ of $X$ with Revuz measure $\delta_{\{a\}}$ and the $W$-valued point process $\mathbf{p}$ associated with $\ell$ defined by (2.11). By Lemma 3.1, $\mathbf{p}$ under 
$\mathbf{P}_{a}$ is an absorbed Poisson point process and admits the representation (3.3) in terms of a $W$-valued Poisson point process $\widetilde{\mathbf{p}}$ defined on some probability space $(\widetilde{\Omega}, \widetilde{\mathbf{P}})$ together with its hitting time $\widetilde{T}$ of $W^{-} \cup\{\partial\}$.

Let $\mathbf{n}$ be the characteristic measure of $\widetilde{\mathbf{p}}$. Then, for any non-negative predictable process $\{a(t, w, \widetilde{\omega}), t \geq 0, w \in W, \widetilde{\omega} \in \widetilde{\Omega}\}$, we have

$$
\widetilde{\mathbf{E}}\left[\sum_{s \leq t} a\left(s, \widetilde{\mathbf{p}}_{s}, \widetilde{\omega}\right)\right]=\widetilde{\mathbf{E}}\left[\int_{W \times(0, t]} a(s, w, \widetilde{\omega}) \mathbf{n}(d w) d s\right],
$$

because the compensator of $\widetilde{\mathbf{p}}$ equals $t \mathbf{n}(\cdot)$ ( cf. [23, §II.3]).

We now proceed along the same line as in [16, Remark 4.2]. The terminal time of $w \in W$ is denoted by $\zeta(w)$ : for $w=k_{\sigma_{a}}(\omega)$ with $\omega \in \Omega, \zeta(w)=\sigma_{a}(\omega)$. We put for $f \in \mathcal{B}^{+}\left(E_{0}\right)$

$$
\check{f}_{\alpha}(w)=\int_{0}^{\zeta(w)} e^{-\alpha t} f((w(t)) d t, \quad w \in W, \quad \alpha>0 .
$$

Note that $t \mapsto X_{t}(\omega)$ has only at most countably many discontinuous points. Thus by (2.2) and the condition (4), $M(\omega)$ has zero Lebesgue measure almost surely. So we have $\mathbf{P}_{a}$-a.s.

$$
\begin{aligned}
\int_{0}^{\infty} e^{-\alpha t} f\left(X_{t}\right) d t & =\sum_{s<\ell_{\infty}} \int_{\tau_{s-}}^{\tau_{s}} e^{-\alpha t} f\left(X_{t}\right) d t+\int_{\tau_{\ell_{\infty}-}}^{\infty} e^{-\alpha t} f\left(X_{t}\right) d t \\
& =\sum_{s<\ell_{\infty}} e^{-\alpha \tau_{s-}} \check{f}_{\alpha}\left(\mathbf{p}_{s}\right)+e^{-\alpha \tau_{L_{\infty}-}} \check{f}_{\alpha}\left(\mathbf{p}_{\ell_{\infty}}\right),
\end{aligned}
$$

which is equivalent in law to

$$
\sum_{s<\widetilde{T}} e^{-\alpha S(s-)} \check{f}_{\alpha}\left(\widetilde{\mathbf{p}}_{s}^{+}\right)+e^{-\alpha S(\tilde{T}-)} \check{f}_{\alpha}\left(\widetilde{\mathbf{p}}_{\widetilde{T}}\right), \quad \text { under } \widetilde{\mathbf{P}},
$$

where $\left\{\widetilde{\mathbf{p}}_{s}^{+}, s>0\right\}$ is a Poisson point process defined by $\widetilde{\mathbf{p}}_{s}^{+}=\widetilde{\mathbf{p}}_{s}$ for $s \in$ $\mathcal{D}_{\widetilde{\mathbf{p}}^{+}}=\left\{s \in \mathcal{D}_{\widetilde{\mathbf{p}}}: \widetilde{\mathbf{p}}_{s} \in W^{+}\right\}$and $S(s)=\sum_{r \leq s} \zeta\left(\widetilde{\mathbf{p}}_{r}^{+}\right)$. The characteristic measure of $\left\{\widetilde{\mathbf{p}}_{s}^{+}, s>0\right\}$ is the restriction $\mathbf{n}^{+}$of $\mathbf{n}$ on $W^{+}$.

First we claim that

$$
\widetilde{\mathbf{E}}\left[e^{-\alpha S(s)}\right]=\exp \left(-\alpha\left(\widehat{u}_{\alpha}, \varphi\right) s\right) .
$$

Since

$$
e^{-\alpha S(s)}-1=\sum_{r \leq s}\left\{e^{-\alpha S(r)}-e^{-\alpha S(r-)}\right\}=\sum_{r \leq s} e^{-\alpha S(r-)}\left\{e^{-\alpha \zeta\left(\mathbf{p}_{r}^{+}\right)}-1\right\},
$$

it follows from (4.15) that

$$
\widetilde{\mathbf{E}}\left[e^{-\alpha S(s)}\right]-1=-c \int_{0}^{s} \widetilde{\mathbf{E}}\left[e^{-\alpha S(r)}\right] d r
$$


with

$$
\begin{aligned}
c & =\mathbf{n}^{+}\left(1-e^{-\alpha \zeta}\right)=\mathbf{n}\left(1-e^{-\alpha \zeta} ; \zeta<\infty\right)=\mathbf{n}\left\{\alpha \int_{0}^{\zeta} e^{-\alpha t} d t ; \zeta<\infty\right\} \\
& =\alpha \int_{0}^{\infty} e^{-\alpha t} \mathbf{n}(t<\zeta<\infty) d t .
\end{aligned}
$$

Due to (3.5) (see also (2.8)), we have accordingly

$$
c=\alpha \int_{0}^{\infty} e^{-\alpha t} \nu_{t}(\varphi) d t=\alpha\left(\widehat{u}_{\alpha}, \varphi\right)
$$

which is finite by Proposition 4.1(v). The identity (4.18) then follows.

On the other hand, we have from Theorem 3.2 and the basic properties of Poisson point processes,

(i) $\widetilde{T}$ has an exponential distribution with exponent $L^{(0)}\left(\widehat{\varphi} \cdot m_{0}, 1-\varphi\right)+\delta_{0}$, where $\delta_{0}$ is defined by (3.6).

(ii) The three objects $\left\{\widetilde{\mathbf{p}}_{s}^{+}, s>0\right\}, \widetilde{T}$ and $\widetilde{\mathbf{p}}_{\widetilde{T}}$ are independent.

(iii) The law of $\widetilde{\mathbf{p}}_{\widetilde{T}}$ is $\overline{\mathbf{n}}^{-}\left(W^{-} \cup\{\partial\}\right)^{-1} \overline{\mathbf{n}}^{-}=\left(L^{(0)}\left(\widehat{\varphi} \cdot m_{0}, 1-\varphi\right)+\delta_{0}\right)^{-1} \overline{\mathbf{n}}^{-}$, where $\overline{\mathbf{n}}^{-}$is the restriction of $\mathbf{n}$ on $W^{-} \cup\{\partial\}$.

Taking these facts and formula (4.15) for $\widetilde{\mathbf{p}}^{+}$into account, we get from (4.16),(4.17) and (4.18),

$$
\begin{aligned}
& G_{\alpha} f(a)=\widetilde{\mathbf{E}}\left[\sum_{s<\widetilde{T}} e^{-\alpha S(s-)} \check{f}_{\alpha}\left(\widetilde{\mathbf{p}}_{s}^{+}\right)+e^{-S\left(\widetilde{T}^{-}\right)} \check{f}_{\alpha}\left(\widetilde{\mathbf{p}}_{\widetilde{T}}\right)\right] \\
= & \widetilde{\mathbf{E}}\left[\int_{0}^{\widetilde{T}} e^{-\alpha\left(\widehat{u}_{\alpha}, \varphi\right) s} d s\right] \mathbf{n}^{+}\left(\check{f}_{\alpha}\right) \\
& +\widetilde{\mathbf{E}}\left(e^{-\alpha\left(\widehat{u}_{\alpha}, \varphi\right) \widetilde{T}}\right)\left(L^{(0)}\left(\widehat{\varphi} \cdot m_{0}, 1-\varphi\right)+\delta_{0}\right)^{-1} \mathbf{n}^{-}\left(\check{f}_{\alpha}\right) \\
= & \frac{\mathbf{n}^{+}\left(\check{f}_{\alpha}\right)}{\alpha\left(\widehat{u}_{\alpha}, \varphi\right)+L^{(0)}\left(\widehat{\varphi}^{\prime} \cdot m_{0}, 1-\varphi\right)+\delta_{0}}+\frac{\mathbf{n}^{-}\left(\check{f}_{\alpha}\right)}{\alpha\left(\widehat{u}_{\alpha}, \varphi\right)+L^{(0)}\left(\widehat{\varphi}^{\prime} \cdot m_{0}, 1-\varphi\right)+\delta_{0}} \\
= & \frac{\mathbf{n}\left(\check{f}_{\alpha}\right)}{\alpha\left(\widehat{u}_{\alpha}, \varphi\right)+L^{(0)}\left(\widehat{\varphi} \cdot m_{0}, 1-\varphi\right)+\delta_{0}},
\end{aligned}
$$

which coincides with the right hand side of (4.11) because we have from Theorem 3.2

$$
\mathbf{n}\left(\check{f}_{\alpha}\right)=\int_{0}^{\infty} e^{-\alpha t} \nu_{t}(f) d t=\left(\widehat{u}_{\alpha}, f\right) .
$$

(4.13) can be obtained analogously.

Under the weak duality assumption (1), the denominators of (4.11) and (4.13) must be equal. Since $\left(\widehat{u}_{\alpha}, \varphi\right)=\left(u_{\alpha}, \widehat{\varphi}\right)$ (see the first two equations in the proof of Lemma 5.8), we must have the identity (4.10). 
In the above proof, we did not use the property of $X$ having no jumps from the point $a$ to $E_{0}$, which is proved in Proposition 4.1(iii). But this property reflects on the following property of the characteristic measure $\mathbf{n}$ of the absorbed Poisson point process $\mathbf{p}$ considered in the above proof.

Proposition 4.4

$$
\mathbf{n}\{w(0) \neq a\}=0 .
$$

Proof. By (4.5), we have $\mathbf{E}_{a}\left(\sum_{s \in G} 1_{\Lambda} \circ i_{s}\right)=0$ for $\Lambda=\{w(0) \neq a\}$ and we get $\mathbf{n}(\Lambda)=\mathbf{Q}^{*}(\Lambda)=0$ from $(2.6)$ and (3.4).

Remark 2. In this section, we have assumed that $E$ is a locally compact separable metric space. But all assertions in this section remain valid for a general Lusin space $E$ except that the identities (4.6), (4.12), (4.14) hold only for q.e. $x \in E_{0}$ rather than for every $x \in E_{0}$, because we need to replace the usage of $[2, \mathrm{p} 59]$ by $[21,(15.7)]$ in the proof of (4.6). The uniqueness statement in Corollary 4.3 should be modified accordingly in the Lusin space case.

We also note that the expression (4.11) of the resolvent has been obtained in [12] by a different method for a general right process $X$ and its excessive measure $m$, in which case $\widehat{X}$ can be taken to be a dual moderate Markov process. But the present proof is more useful in the next section.

\section{Extending Markov process via Poisson point processes of excursions}

As in $\S 4$, let $E$ be a locally compact separable metric space and $a$ be a fixed non-isolated point of $E$ and $m_{0}$ be a $\sigma$-finite measure on $E_{0}:=E \backslash\{a\}$ with $\operatorname{Supp}\left[m_{0}\right]=E$. We extend $m_{0}$ to a measure $m$ on $E$ by setting $m(\{a\})=0$. Note that $m$ could be infinity on a compact neighborhood of $a$ in $E$. Let $E_{\Delta}=E \cup\{\Delta\}$ be the one point compactification of $E$. When $E$ is compact, $\Delta$ is added as an isolated point.

\subsection{Excursion laws in duality}

We shall assume that we are given two Borel standard processes $X^{0}=$ $\left\{X_{t}^{0}, \mathbf{P}_{x}^{0}, \zeta^{0}\right\}$ and $\widehat{X}^{0}=\left\{\widehat{X}_{t}^{0}, \widehat{\mathbf{P}}_{x}^{0}, \widehat{\zeta}^{0}\right\}$ on $E_{0}$ satisfying the following conditions.

(A.1) $X^{0}$ and $\widehat{X}^{0}$ are in weak duality with respect to $m_{0}$, that is, for every $\alpha>0$, and $f, g \in \mathcal{B}^{+}\left(E_{0}\right)$,

$$
\int_{E_{0}} \widehat{G}_{\alpha}^{0} f(x) g(x) m_{0}(d x)=\int_{E_{0}} f(x) G_{\alpha}^{0} g(x) m_{0}(d x),
$$

where $G_{\alpha}^{0}$ and $\widehat{G}_{\alpha}^{0}$ are the resolvents of $X^{0}$ and $\widehat{X}^{0}$, respectively. 
(A.2) $X^{0}$ and $\widehat{X}^{0}$ satisfy, for every $x \in E_{0}$,

$$
\begin{aligned}
& \mathbf{P}_{x}^{0}\left(\zeta^{0}<\infty, X_{\zeta^{0}-}^{0}=a\right)>0, \\
& \mathbf{P}_{x}^{0}\left(\zeta^{0}<\infty, X_{\zeta^{0}-}^{0} \in\{a, \Delta\}\right)=\mathbf{P}_{x}^{0}(\zeta<\infty), \\
& \widehat{\mathbf{P}}_{x}^{0}\left(\widehat{\zeta}^{0}<\infty, \widehat{X}_{\widehat{\zeta}^{0}-}^{0}=a\right)>0, \\
& \widehat{\mathbf{P}}_{x}^{0}\left(\widehat{\zeta}^{0}<\infty, \widehat{X}_{\widehat{\zeta}^{0}-}^{0} \in\{a, \Delta\}\right)=\widehat{\mathbf{P}}_{x}^{0}(\widehat{\zeta}<\infty) . x
\end{aligned}
$$

Here, as in $\S 4$, for a Borel set $B \subset \mathbf{E}_{\Delta}$, the notation " $X_{\zeta^{0}-}^{0} \in B$ " means that the left limit of $t \mapsto X_{t}^{0}$ at $t=\zeta^{0}$ exists under the topology of $E_{\Delta}$ and takes values in $B$.

The first condition in (5.1) (resp. (5.2)) means that $X^{0}$ (resp. $\left.\widehat{X}^{0}\right)$ is approachable to the point $a$, while the second condition in (5.1) (resp. (5.2)) implies that $X^{0}$ (resp. $\widehat{X}^{0}$ ) admits no killings inside $E_{0}$.

As in $\S 4$, we put for $x \in E_{0}$ and $\alpha>0$,

$$
\varphi(x):=\mathbf{P}_{x}^{0}\left(\zeta^{0}<\infty, X_{\zeta^{0}-}^{0}=a\right) \quad \text { and } \quad u_{\alpha}(x):=\mathbf{E}_{x}^{0}\left[e^{-\alpha \zeta^{0}} ; X_{\zeta^{0}-}^{0}=a\right] .
$$

The corresponding notations for $\widehat{X}^{0}$ will be designated by $\widehat{\varphi}$ and $\widehat{u}_{\alpha}$. As in $\S 2$, the $X^{0}$-energy functional $L^{(0)}\left(\widehat{\varphi} \cdot m_{0}, v\right)$ of the $X^{0}$-excessive measure $\widehat{\varphi} \cdot m_{0}$ and an $X^{0}$-excessive function $v$ is well defined. Similarly the $\widehat{X}^{0}$-energy functional $\widehat{L}^{(0)}\left(\varphi \cdot m_{0}, \widehat{v}\right)$ is well defined. The inner product of $u, v$ in $L^{2}\left(E_{0}, m_{0}\right)$ will be denoted by $(u, v)$, that is, $(u, v)=\int_{E_{0}} u(x) v(x) m_{0}(d x)$. The space of all bounded continuous functions on $E_{0}$ will be denoted by $C_{b}\left(E_{0}\right)$.

We impose some more assumptions:

(A.3) $u_{\alpha}, \widehat{u}_{\alpha} \in L^{1}\left(E_{0}, m_{0}\right)$ for every $\alpha>0$.

(A.4) $G^{0} f(x), \widehat{G}^{0} f(x), x \in E_{0}$, are lower semi-continuous for any Borel $f \geq 0$. Here $G^{0}$ denotes the 0 -order resolvent of $X^{0}$ :

$$
G^{0} f(x):=\mathbf{E}_{x}\left[\int_{0}^{\infty} f\left(X_{t}\right) d t\right]=\uparrow \lim _{\alpha \downarrow 0} G_{\alpha}^{0} f(x)
$$

for $x \in E$ and Borel function $f \geq 0$ on $E$. The 0 -order resolvent $\widehat{G}^{0}$ of $\widehat{X}^{0}$ is similarly defined.

We note that, if $G_{\alpha}^{0}\left(C_{b}\left(E_{0}\right)\right) \subset C_{b}\left(E_{0}\right), \widehat{G}_{\alpha}^{0}\left(C_{b}\left(E_{0}\right)\right) \subset C_{b}\left(E_{0}\right), \alpha>0$, then (A.4) is satisfied by the monotone class lemma.

The next condition will be imposed only when $X^{0}$ is non-symmetric, namely, when $X^{0} \neq \widehat{X}^{0}$.

(A.5) $\lim _{x \rightarrow a} u_{\alpha}(x)=\lim _{x \rightarrow a} \widehat{u}_{\alpha}(x)=1$, for every $\alpha>0$. 
The next condition (A.6) will be imposed only when $X^{0}$ is not a diffusion, namely, when

$$
\mathbf{P}_{m}^{0}\left(X_{t-}^{0} \neq X_{t}^{0} \quad \text { for some } t \in\left(0, \zeta^{0}\right)\right)>0 \text {. }
$$

Note that $\widehat{X}^{0}$ then has the same property in view of $[21, \S 10]$. According to $[31,(73.1),(47.10)]$, the standard process $X^{0}$ on $E_{0}$ has a Lévy system $(N, H)$ on $E_{0}$. That is, $N(x, d y)$ is a kernel on $\left(E_{0}, \mathcal{B}\left(E_{0}\right)\right)$ and $H$ is a PCAF of $X^{0}$ in the strict sense with bounded 1-potential such that for any nonnegative Borel function $f$ on $E_{0} \times\left(E_{0} \cup\left\{\Delta_{0}\right\}\right)$ that vanishes on the diagonal and is extended to be zero outside $E_{0} \times E_{0}$,

$$
\mathbf{E}_{x}^{0}\left[\sum_{s \leq t} f\left(X_{s-}^{0}, X_{s}^{0}\right)\right]=\mathbf{E}_{x}^{0}\left[\int_{0}^{t} \int_{E_{0}} f\left(X_{s}^{0}, y\right) N\left(X_{s}^{0}, d y\right) d H_{s}\right]
$$

for every $x \in E_{0}$ and $t \geq 0$. Similarly, the standard process $\widehat{X}^{0}$ has a Lévy system $(\widehat{N}, \widehat{H})$. Let $\mu_{H}$ and $\mu_{\widehat{H}}$ be the Revuz measure of the PCAF $H$ of $X^{0}$ and the PCAF $\widehat{H}$ of $\widehat{X}^{0}$ with respect to the measure $m_{0}$ on $E_{0}$, respectively. Define

$$
J_{0}(d x, d y):=N(x, d y) \mu_{H}(d x) \quad \text { and } \quad \widehat{J}_{0}(d x, d y):=\widehat{N}(x, d y) \mu_{\widehat{H}}(d x) .
$$

The measures $J_{0}$ and $\widehat{J}_{0}$ are called the jumping measure of $X^{0}$ and $\widehat{X}^{0}$, respectively. It is known (see [18]) that

$$
J_{0}(d x, d y)=\widehat{J}_{0}(d y, d x) \quad \text { on } E_{0} \times E_{0} .
$$

We now state the condition (A.6).

(A.6) Either $E \backslash U$ is compact for any neighborhood $U$ of $a$ in $E$, or for any open neighborhood $U_{1}$ of $a$ in $E$, there exists an open neighborhood $U_{2}$ of $a$ in $E$ with $\bar{U}_{2} \subset U_{1}$ such that

$$
J_{0}\left(U_{2} \backslash\{a\}, E_{0} \backslash U_{1}\right)<\infty \quad \text { and } \quad \widehat{J}_{0}\left(U_{2} \backslash\{a\}, E_{0} \backslash U_{1}\right)<\infty .
$$

Throughout this section, we assume that we are given a pair of Borel standard processes $X^{0}$ and $\widehat{X}^{0}$ on $E_{0}$ satisfying conditions (A.1), (A.2), (A.3), (A.4), and additionally (A.5) in non-symmetric case and (A.6) in non-diffusion case. We aim at constructing (see Theorem 5.15) under these conditions their right process extensions $X, \widehat{X}$ to $E$ with resolvents (4.11), (4.13) respectively. Theorem 5.16 will then be concerned with some stronger conditions (A.1)' and (A.4)' to ensure the quasi-left continuity of the constructed processes so that they become standard.

We note that, if $X^{0}$ is an $m_{0}$-symmetric diffusion on $E_{0}$, then the present conditions (A.2), (A.3) are the same as the conditions A.1, A.2, A.3 assumed in $[16, \S 4]$, while the present (A.4) is weaker than A.4 of [16, §4] as 
is noted in the paragraph below (A.4). Therefore the results of this paper extend the construction problem treated in $[16, \S 4]$ to a more general case. However we shall proceed along the same line as was laid in $[16, \S 4]$.

In Theorem 5.17 at the end of this section, we shall present a stronger variant (A.2)' of the condition (A.2) and prove using a time change argument that, under the conditions (A.1), (A.2)', (A.4) and additionally (A.5) in non-symmetric case and (A.6) in non-diffusion case, the integrability condition (A.3) holds automatically and therefore can be dropped.

As is shown in [5, Lemma 3.1], the measure $\widehat{\varphi} \cdot m_{0}$ is $X^{0}$-purely excessive and accordingly there exists a unique entrance law $\left\{\mu_{t}\right\}_{t>0}$ for $X^{0}$ characterized by

$$
\widehat{\varphi} \cdot m_{0}=\int_{0}^{\infty} \mu_{t} d t .
$$

Analogously there exists a unique $\widehat{X}^{0}$-entrance law $\left\{\widehat{\mu}_{t}\right\}_{t>0}$ characterized by

$$
\varphi \cdot m_{0}=\int_{0}^{\infty} \widehat{\mu}_{t} d t
$$

Further by [5, Lemma 3.1], the Laplace transforms of $\mu_{t}, \widehat{\mu}_{t}$ satisfy

$$
\int_{0}^{\infty} e^{-\alpha t}\left\langle\mu_{t}, f\right\rangle d t=\left(\widehat{u}_{\alpha}, f\right) \quad \text { and } \quad \int_{0}^{\infty} e^{-\alpha t}\left\langle\widehat{\mu}_{t}, f\right\rangle d t=\left(u_{\alpha}, f\right)
$$

for every $\alpha>0$ and $f \in \mathcal{B}^{+}\left(E_{0}\right)$. On account of the assumption (A.3), we then have that for every $t>0$,

$\mu_{t}\left(E_{0}\right)<\infty, \widehat{\mu}_{t}\left(E_{0}\right)<\infty, \quad$ and $\quad \int_{0}^{1} \mu_{s}\left(E_{0}\right) d s<\infty, \quad \int_{0}^{1} \widehat{\mu}_{s}\left(E_{0}\right) d s<\infty$.

We now introduce the spaces $W^{\prime}$ and $W$ of excursions by

$$
\begin{aligned}
W^{\prime} & =\left\{w: \text { a cadlag function from }(0, \zeta(w)) \text { to } E_{0} \text { for some } \zeta(w) \in(0, \infty]\right\}, \\
W & \left.=\left\{w \in W^{\prime}: \text { if } \zeta(w)<\infty \text { then } w(\zeta(w)-):=\lim _{t \uparrow \zeta(\omega)} w(t) \in\{a, \Delta\}\right\} 5.11\right)
\end{aligned}
$$

We call $\zeta(w)$ the terminal time of the excursion $w$.

We are concerned with a measure $\mathbf{n}$ on the space $W$ specified in terms of the entrance law $\left\{\mu_{t}, t>0\right\}$ and the transition semigroup $\left\{P_{t}^{0}, t \geq 0\right\}$ of $X^{0}$ by

$$
\begin{aligned}
& \int_{W} f_{1}\left(w\left(t_{1}\right)\right) f_{2}\left(w\left(t_{2}\right)\right) \cdots f_{n}\left(w\left(t_{n}\right)\right) \mathbf{n}(d w)=\mathbf{E}_{\mu_{t_{1}}}\left[\prod_{k=1}^{n} f_{k}\left(X_{t_{k}-t_{1}}^{0}\right)\right] \\
= & \mu_{t_{1}} f_{1} P_{t_{2}-t_{1}}^{0} f_{2} \cdots P_{t_{n-1}-t_{n-2}}^{0} f_{n-1} P_{t_{n}-t_{n-1}}^{0} f_{n},
\end{aligned}
$$

for any $0<t_{1}<t_{2}<\cdots<t_{n}, f_{1}, f_{2}, \cdots, f_{n} \in B_{b}\left(E_{0}\right)$. Here, we use the convention that $w \in W$ satisfies $w(t):=\Delta$ for $w \in W$ and $t \geq \zeta(w)$, and any 
function $f$ on $E_{0}$ is extended to $E_{0} \cup \Delta$ by setting $f(\Delta)=0$. Further, on the right hand side of (5.12), we employ an abbreviated notation for the repeated operations

$$
\mu_{t_{1}}\left(f_{1} P_{t_{2}-t_{1}}^{0}\left(f_{2} \cdots P_{t_{n-1}-t_{n-2}}^{0}\left(f_{n-1} P_{t_{n}-t_{n-1}}^{0} f_{n}\right) \cdots\right)\right) .
$$

Proposition 5.1 There exists a unique measure $\mathbf{n}$ on the space $W$ satisfying (5.12).

Proof. Let $\mathbf{n}$ be the Kuznetsov measure on $W^{\prime}$ uniquely associated with the transition semigroup $\left\{P_{t}^{0}, t \geq 0\right\}$ and the entrance rule $\left\{\eta_{u}, u \in \mathbb{R}\right\}$ defined by

$$
\eta_{u}=0 \quad \text { for } u \leq 0 \quad \text { and } \quad \eta_{u}=\mu_{u} \quad \text { for } u>0,
$$

as is constructed in [8, Chap XIX, §9] for a right semigroup. Because of the present choice of the entrance rule, it holds that the random birth time $\alpha$ for the Kuznetsov process is identically 0 (cf. [20, p54]).

On account of the assumption (A.2) for the standard process $X^{0}$ on $E_{0}$, the same method of the construction of the Kuznetsov measure as in $[8$, Chap.XIX, 9] works in proving that $\mathbf{n}$ is carried on the space $W$ and satisfies (5.12).

We call $\mathbf{n}$ the excursion law associated with the entrance law $\left\{\mu_{t}\right\}$ for $X^{0}$. It is strong Markov with respect to the transition semigroup $\left\{P_{t}^{0}, t \geq 0\right\}$ of $X^{0}$. Analogously we can introduce the excursion law $\widehat{\mathbf{n}}$ on the space $W$ associated with the entrance law $\widehat{\mu}_{t}$ for $\widehat{X}^{0}$.

We split the space $W$ of excursions into two parts:

$$
W^{+}:=\{w \in W: \zeta(w)<\infty \text { and } w(\zeta-)=a\} \quad \text { and } \quad W^{-}:=W \backslash W^{+} .
$$

For $w \in W^{+}$, we define time-reversed path $\widehat{w} \in W^{\prime}$ by

$$
\widehat{w}(t):=w((\zeta-t)-)=\lim _{t^{\prime} \uparrow t} w\left(\zeta-t^{\prime}\right), \quad 0<t<\zeta .
$$

The next lemma asserts that the excursion laws $\mathbf{n}$ and $\widehat{\mathbf{n}}$ restricted to $W^{+}$ are interchangeable under this time reversion.

Lemma 5.2 For any $t_{k}>0$ and $f_{k} \in \mathcal{B}_{b}\left(S_{0}\right),(1 \leq k \leq n)$,

$$
\begin{aligned}
& \mathbf{n}\left\{\prod_{k=1}^{n} f_{k}\left(w\left(t_{1}+\cdots+t_{k}\right)\right) ; W^{+}\right\}=\mu_{t_{1}} f_{1} P_{t_{2}}^{0} f_{2} \cdots P_{t_{n-1}}^{0} f_{n-1} P_{t_{n}}^{0} f_{n} \varphi, \\
& \mathbf{n}\left\{\prod_{k=1}^{n} f_{k}\left(w\left(t_{1}+\cdots+t_{k}\right)\right) ; W^{+}\right\}=\widehat{\mathbf{n}}\left\{\prod_{k=1}^{n} f_{k}\left(\widehat{w}\left(t_{1}+\cdots+t_{k}\right)\right) ; W^{+}\right\} .
\end{aligned}
$$


Proof. (5.15) readily follows from (5.12) and the Markov property of $\mathbf{n}$. As for (5.16), we observe that, for $\alpha_{1}, \cdots, \alpha_{n}>0$,

$$
\begin{aligned}
& \int_{0}^{\infty} \cdots \int_{0}^{\infty} e^{\left.-\sum\right) k=1^{n} \alpha_{k}} \mathbf{n}\left\{\prod_{k=1}^{n} f_{k}\left(w\left(t_{1}+\cdots+t_{k}\right)\right) ; W^{+}\right\} d t_{1} \cdots d t_{n} \\
= & \mathbf{n}\{F(w) ; \zeta<\infty, w(\zeta-)=a\},
\end{aligned}
$$

where, with $t+0:=0$

$$
F(w)=n ! \int_{0<t_{1}<\cdots<t_{n}<\zeta} \prod_{k=1}^{n}\left\{e^{-\alpha_{k}\left(t_{k}-t_{k-1}\right)} f_{k}\left(w\left(t_{k}\right)\right)\right\} d t_{1} \cdots d t_{n} .
$$

Hence, for (5.16), it suffices to prove for $f_{k} \in C_{b}\left(E_{0}\right), 1 \leq k \leq n$,

$$
\mathbf{n}\{F(w) ; \zeta<\infty, w(\zeta-)=a\}=\widehat{\mathbf{n}}\{F(\widehat{w}) ; \zeta<\infty, w(\zeta-)=a\} .
$$

Changing of variables $\zeta-t_{k}=s_{k}$ for $0 \leq k \leq n$ in the following expression

$$
F(\widehat{w})=n ! \int_{0<t_{1}<\cdots<t_{n}<\zeta} \prod_{k=1}^{n}\left\{e^{-\alpha_{k}\left(t_{k}-t_{k-1}\right)} f_{k}\left(w\left(\left(\zeta-t_{k}\right)-\right)\right)\right\} d t_{1} \cdots d t_{n},
$$

where $t_{0}:=0$, and noting that

$$
s_{0}=\zeta \text { and } 0<t_{1}<\cdots<t_{n}<\zeta \text { if and only if } 0<s_{n}<\cdots<s_{1}<\zeta,
$$

we obtain

$$
\begin{aligned}
F(\widehat{w}) & =n ! \int_{0<s_{n}<\cdots<s_{1}<\zeta} \prod_{k=1}^{n}\left\{e^{-\alpha_{k}\left(s_{k-1}-s_{k}\right)} f_{k}\left(w\left(s_{k}\right)\right)\right\} d s_{1} \cdots d s_{n} \\
& =n ! \int_{0<s_{n}<\cdots<s_{1}<\infty} \Gamma_{s_{1} \cdots s_{n}}(w) d s_{1} \cdots d s_{n},
\end{aligned}
$$

where

$$
\Gamma_{s_{1} \cdots s_{n}}(w)=\prod_{k=2}^{n}\left\{e^{-\alpha_{k}\left(s_{k-1}-s_{k}\right)} f_{k}\left(w\left(s_{k}\right)\right)\right\} \cdot e^{-\alpha_{1}\left(\zeta-s_{1}\right)} f_{1}\left(w\left(s_{1}\right)\right) 1_{(0, \zeta)}\left(s_{1}\right) .
$$

On the other hand, we get from (5.10) and the Markov property of $\widehat{\mathbf{n}}$ that

$$
\begin{aligned}
& \widehat{\mathbf{n}}\left\{\Gamma_{s_{1} s_{2} \cdots s_{n}}(w) ; \zeta<\infty, w(\zeta-)=a\right\} \\
= & \widehat{\mathbf{n}}\left\{f _ { n } ( w ( s _ { n } ) ) e ^ { - \alpha _ { n } ( s _ { n - 1 } - s _ { n } ) } \cdots f _ { 2 } \left(\left(w\left(s_{2}\right)\right) e^{-\alpha_{2}\left(s_{1}-s_{2}\right)}\right.\right. \\
\left.f_{1}\left(w\left(s_{1}\right)\right) u_{\alpha_{1}}\left(w\left(s_{1}\right)\right) ; s_{1}<\zeta\right\} & e^{-\sum_{k=2}^{n} \alpha_{k}\left(s_{k-1}-s_{k}\right.} \widehat{\mu}_{s_{n}} f_{n} \widehat{P}_{s_{n-1}-s_{n}}^{0} f_{n-1} \widehat{P}_{s_{n-2}-s_{n-1}}^{0} f_{n-1} \\
& \cdots \widehat{P}_{s_{2}-s_{3}}^{0} f_{2} \widehat{P}_{s_{1}-s_{2}}^{0} f_{1} \widehat{u}_{\alpha_{1}} .
\end{aligned}
$$


Therefore,

$$
\begin{aligned}
& \widehat{\mathbf{n}}\{F(\widehat{w}) ; \zeta<\infty, w(\zeta-)=a\} \\
= & \int_{0}^{\infty} d s_{n} \widehat{\mu}_{s_{n}} f_{n} \widehat{G}_{\alpha_{n}}^{0} f_{n-1} \widehat{G}_{\alpha_{n-1}}^{0} \cdots f_{3} \widehat{G}_{\alpha_{3}}^{0} f_{2} \widehat{G}_{\alpha_{2}}^{0} f_{1} \widehat{u}_{\alpha_{1}} .
\end{aligned}
$$

In view of (5.8), the weak duality (A.1), (5.15) and (5.17), we arrive at

$$
\begin{aligned}
\widehat{\mathbf{n}}\{F(\widehat{w}) ; & \zeta<\infty, w(\zeta-)=a\} \\
= & \left\langle\varphi \cdot m_{0}, f_{n} \widehat{G}_{\alpha_{n}}^{0} f_{n-1} \widehat{G}_{\alpha_{n-1}}^{0} \cdots f_{3} \widehat{G}_{\alpha_{3}}^{0} f_{2} \widehat{G}_{\alpha_{2}}^{0} f_{1} \widehat{u}_{\alpha_{1}}\right\rangle \\
= & \left(f_{n} \varphi, \widehat{G}_{\alpha_{n}}^{0} f_{n-1} \widehat{G}_{\alpha_{n-1}}^{0} \cdots f_{3} \widehat{G}_{\alpha_{3}}^{0} f_{2} \widehat{G}_{\alpha_{2}}^{0} f_{1} \widehat{u}_{\alpha_{1}}\right) \\
= & \left(f_{1} G_{\alpha_{2}}^{0} f_{2} G_{\alpha_{3}}^{0} f_{3} \cdots G_{\alpha_{n}}^{0} f_{n} \varphi, \widehat{u}_{\alpha_{1}}\right) \\
= & \int_{0}^{\infty} e^{-\alpha_{1} t_{1}} \mu_{t_{1}} f_{1} G_{\alpha_{2}}^{0} f_{2} G_{\alpha_{3}}^{0} f_{3} \cdots G_{\alpha_{n}}^{0} f_{n} \varphi d t_{1} \\
= & \mathbf{n}\{F(w) ; \zeta<\infty \text { and } w(\zeta-)=a\},
\end{aligned}
$$

the desired identity (5.18). This establishes (5.16).

Next we define

$$
W_{a}:=\left\{w \in W: w(0+):=\lim _{t \downarrow 0} w(t)=a\right\} .
$$

Lemma 5.3 $\mathbf{n}\left\{W \backslash W_{a}\right\}=0$ and $\widehat{\mathbf{n}}\left\{W \backslash W_{a}\right\}=0$.

Proof. The preceding lemma implies that

$$
\begin{aligned}
\mathbf{n}\left\{W^{+} \backslash W_{a}\right\} & =\mathbf{n}\left\{W^{+} \cap(w(0+)=a)^{c}\right\} \\
& =\widehat{\mathbf{n}}\left\{W^{+} \cap(\widehat{w}(0+)=a)^{c}\right\} \\
& =\widehat{\mathbf{n}}\left\{W^{+} \cap(w(\zeta-)=a)^{c}\right\} \\
& =0 .
\end{aligned}
$$

We then have for each $t>0$

$$
\mathbf{n}\left\{\varphi(w(t)) ;(\zeta>t) \cap(w(0+)=a)^{c}\right\}=\mathbf{n}\left\{\left(W^{+} \backslash W_{a}\right) \cap(\zeta>t)\right\}=0 .
$$

As $\varphi(x)>0$ for every $x \in E_{0}$ by the assumption (A.2). we conclude that

$$
\mathbf{n}\left\{\left(W \backslash W_{a}\right) \cap(\zeta>t)\right\}=0 \quad \text { for every } t>0,
$$

and therefore $\mathbf{n}\left\{\left(W \backslash W_{a}\right)\right\}=0$ after letting $t \downarrow 0$. The same property of $\widehat{\mathbf{n}}$ can be shown analogously. 
Lemma 5.4 For any neighborhood $U$ of a in $E$, define

$$
\tau_{U}(w)=\inf \{t>0: w(t) \notin U\} \quad \text { for } w \in W .
$$

Then

$$
\mathbf{n}\left\{\tau_{U}<\zeta\right\}<\infty \quad \text { and } \quad \widehat{\mathbf{n}}\left\{\tau_{U}<\zeta\right\}<\infty .
$$

Proof. We only give a proof for $\mathbf{n}$. Let $V$ be any neighborhood of $a$ in $E$. It suffices to show

$$
\mathbf{n}\left(\tau_{U}<\zeta\right)<\infty
$$

for some neighborhood $U$ of $a$ with $U \subset V$. We choose such $U$ as follows. Let us fix a relatively compact open neighborhood $U_{1}$ of $a$ in $E$. When $X^{0}$ is a diffusion, we put $U=V \cap U_{1}$. When $X^{0}$ is not a diffusion and the second condition of (A.5) is fulfilled, we take $U_{2}$ in the condition for $U_{1}$ and put $U=V \cap U_{2}$.

By virtue of the relation

$$
\varphi-u_{1}=G_{1}^{0} \varphi=G^{0} u_{1}
$$

and the assumption (A.4), the function $G_{1}^{0} \varphi$ is lower semi-continuous on $E_{0}$. Furthermore, since $\varphi$ is $X^{0}$-excessive and strictly positive by assumption (A.2), $G_{1}^{0} \varphi$ is moreover strictly positive on $E_{0}$. As $\overline{U_{1}}$ is compact in $E$,

$$
\delta:=\frac{1}{2} \inf _{x \in \bar{U}_{1} \backslash U} G_{1}^{0} \varphi(x)>0 .
$$

Since $G_{1}^{0} \varphi(x)=\int_{0}^{\infty} e^{-t} \mathbf{P}_{x}\left(t<\zeta^{0}<\infty, X_{\zeta^{0}-}^{0}=a\right) d t$, we have

$$
\mathbf{P}_{x}\left(\delta<\zeta^{0}<\infty, X_{\zeta^{0}-}^{0}=a\right)>\delta \quad \text { for every } x \in \bar{U}_{1} \backslash U .
$$

We shall use the notation $\tau_{U}$ not only for $w \in W$ but also for the sample path of the Markov process $X^{0}$. Using the preceding lemma, we have

$$
\mathbf{n}\left\{\tau_{U}<\zeta^{0}\right\}=\lim _{\epsilon \downarrow 0} \mathbf{n}\left\{\epsilon<\tau_{U}<\zeta^{0}\right\}=\lim _{\epsilon \downarrow 0} \int_{U} \mu_{\epsilon}(d x) \mathbf{P}_{x}^{0}\left\{\tau_{U}<\zeta^{0}\right\}=I+I I,
$$

where

$$
\begin{array}{r}
I:=\lim _{\epsilon \downarrow 0} \int_{U} \mu_{\epsilon}(d x) \mathbf{P}_{x}^{0}\left(\tau_{U}<\zeta^{0}, X_{\tau_{U}}^{0} \in \bar{U}_{1} \backslash U\right), \\
I I:=\lim _{\epsilon \downarrow 0} \int_{U} \mu_{\epsilon}(d x) \mathbf{P}_{x}^{0}\left(\tau_{U}<\zeta^{0}, X_{\tau_{U}}^{0} \in E_{0} \backslash U_{1}\right) .
\end{array}
$$

From (5.21) and (5.10), it follows that 


$$
\begin{aligned}
I & \leq \varlimsup_{\epsilon \downarrow 0} \int_{U} \mu_{\epsilon}(d x) \mathbf{E}_{x}^{0}\left[\delta^{-1} \mathbf{P}_{X_{\tau_{U}}^{0}}\left(\delta<\zeta^{0}<\infty, X_{\zeta^{0}-}^{0}=a\right)\right. \\
& \left.\leq \tau_{U}<\zeta^{0}, X_{\tau_{U}}^{0} \in \bar{U}_{1} \backslash U\right] \\
& \leq \delta^{-1} \lim _{\epsilon \downarrow 0} \int_{E_{0}} \mu_{\epsilon}(d x) \mathbf{P}_{x}^{0}\left(\delta<\zeta^{0}<\infty, X_{\zeta^{0}-}^{0}=a\right) \\
& =\delta^{-1} \lim _{\epsilon \downarrow 0} \mu_{\epsilon+\delta}\left(E_{0}\right) \\
& \leq \delta^{-1} \mu_{\delta}\left(E_{0}\right)<\infty .
\end{aligned}
$$

II may not vanish when $X^{0}$ is not a diffusion. In this case, let $(N(x, d y), H)$ be the Lévy system of $X^{0}$ appearing in the condition (A.5). Note that

$$
\begin{aligned}
I I & =\lim _{\epsilon \downarrow 0} \int_{U} \mu_{\epsilon}(d x) \mathbf{E}_{x}^{0}\left[\int_{0}^{\tau_{U}} 1_{U}\left(X_{s}^{0}\right) N\left(X_{s}^{0}, E \backslash U_{1}\right) d H_{s}\right] \\
& \leq \lim _{\epsilon \downarrow 0} \int_{E_{0}} \mu_{\epsilon}(d x) \mathbf{E}_{x}^{0}\left[\int_{0}^{\infty} 1_{U}\left(X_{s}^{0}\right) N\left(X_{s}^{0}, E \backslash U_{1}\right) d H_{s}\right] \\
& =\lim _{\epsilon \downarrow 0} \int_{E_{0}} \mu_{\varepsilon}(d x) G^{0} \mu_{K}(x)
\end{aligned}
$$

where $\mu_{K}(d x):=1_{U}(x) N\left(x, E_{0} \backslash U_{1}\right) \mu_{H}(d x)$ is the Revuz measure of the PCAF of $X^{0}$

$$
K_{t}:=\int_{0}^{t} 1_{U}\left(X_{s}^{0}\right) N\left(X_{s}^{0}, E \backslash U_{1}\right) d H_{s}, \quad t \geq 0,
$$

and $G^{0} \mu_{K}(x):=\mathbf{E}_{x}\left[K_{\infty}\right]$. Note that $\mu_{K}$ is a finite measure on $E_{0}$ by assumption (A.5). For $\alpha>0$ and $x \in E_{0}$, we define

$$
G_{\alpha}^{0} \mu_{K}(x):=\mathbf{E}_{x}\left[\int_{0}^{\infty} e^{-\alpha t} d K_{t}\right] .
$$

Observe that $\alpha G_{\alpha}^{0} G^{0} \mu_{K}$ increases to $G^{0} \mu_{K}$ as $\alpha \uparrow \infty$. We have, by (5.7), the identity $G_{\alpha}^{0} G^{0} \mu_{K}=G^{0} G_{\alpha}^{0} \mu_{K}$ and [21, (9.3)],

$$
\begin{aligned}
\int_{E_{0}} \mu_{\varepsilon}(d x) G^{0} \mu_{K}(x) & =\lim _{\alpha \rightarrow \infty} \alpha \int_{E_{0}} \mu_{\varepsilon}(d x) G^{0} G_{\alpha}^{0} \mu_{K}(x) \\
& =\lim _{\alpha \rightarrow \infty} \int_{0}^{\infty}\left\langle\mu_{\varepsilon} P_{t}^{0}, \alpha G_{\alpha}^{0} \mu_{K}\right\rangle d t \\
& \leq \lim _{\alpha \rightarrow \infty} \int_{0}^{\infty}\left\langle\mu_{t}, \alpha G_{\alpha}^{0} \mu_{K}\right\rangle d t=\lim _{\alpha \rightarrow \infty}\left\langle\widehat{\varphi} \cdot m_{0}, \alpha G_{\alpha}^{0} \mu_{K}\right\rangle \\
& =\lim _{\alpha \rightarrow \infty}\left\langle\alpha \widehat{G}_{\alpha}^{0} \widehat{\varphi}, \mu_{K}\right\rangle=\int_{E_{0}} \widehat{\varphi}(x) \mu_{K}(d x) \leq \mu_{K}\left(E_{0}\right)<\infty
\end{aligned}
$$


Hence we get the desired finiteness of $I I$.

When the first condition of (A.5) is fulfilled, the first half of the preceding proof is enough if we replace $U, \bar{U}_{1}$ with $V, E_{0}$ respectively.

Lemma 5.5 $\mathbf{n}\left(W^{-}\right)=L^{0}\left(\widehat{\varphi} \cdot m_{0}, 1-\varphi\right)<\infty$ and

$$
\widehat{\mathbf{n}}\left(W^{-}\right)=\widehat{L}^{0}\left(\varphi \cdot m_{0}, 1-\widehat{\varphi}\right)<\infty .
$$

Proof. Since $\mathbf{n}\left(\zeta>t ; W^{-}\right)=\left\langle\mu_{t}, 1-\varphi\right\rangle$, the first identity follows from [ 5 , Lemma 3.1] by letting $t \downarrow 0$. Take a relatively compact neighborhood $U$ of $a$ in $E$. Since $a \in E$ and $\Delta$ is a one-point compactification of $E$, we have

$$
\{\zeta<\infty \text { and } w(\zeta-)=\Delta\} \subset\left\{\tau_{U}<\zeta\right\} .
$$

Hence for any $t>0$,

$$
\begin{aligned}
\mathbf{n}\left(W^{-}\right) & =\mathbf{n}\{\zeta<\infty, w(\zeta-)=\Delta\}+\mathbf{n}\{\zeta=\infty\} \\
& \leq \mathbf{n}\left\{\tau_{U}<\zeta\right\}+\mathbf{n}\{\zeta>t\} \\
& =\mathbf{n}\left\{\tau_{U}<\zeta\right\}+\mu_{t}\left(E_{0}\right),
\end{aligned}
$$

which is finite by Lemma 5.4 and (5.10). The second assertion can be shown similarly.

\subsection{Poisson point processes on $W_{a} \cup\{\partial\}$ and a new process $X^{a}$}

By Lemma 5.3, the excursion law $\mathbf{n}$ is concentrated on the space $W_{a}$ defined by (5.19). In correspondence to (5.13), we define

$$
W_{a}^{+}:=\left\{w \in W^{+}: \lim _{t \downarrow 0} w(t)=a\right\} \quad \text { and } \quad W_{a}^{-}:=\left\{w \in W^{-}: \lim _{t \downarrow 0} w(t)=a\right\},
$$

so that $W_{a}=W_{a}^{+}+W_{a}^{-}$. In the sequel however, we shall employ slightly modified but equivalent definitions of those spaces by extending each $w$ from an $E_{0}$-valued excursion to $E$-valued one as follows:

$$
\begin{gathered}
W_{a}=\{w: \text { a cadlag function from }[0, \zeta(w)) \text { to } E \text { for some } \zeta(w) \in(0, \infty] \\
\text { with } w(0)=a, w(t) \in E_{0} \text { for } t \in(0, \zeta(w)) \\
\quad \text { and } w(\zeta(w)-) \in\{a, \Delta\} \text { if } \zeta(w)<\infty\} .
\end{gathered}
$$

Any $w \in W_{a}$ with the properties $\zeta(w)<\infty$ and $w(\zeta(w)-)=a$ will be regarded to be a cadlag function from $[0, \zeta(w)]$ to $E$ by setting $w(\zeta(w))=a$. We further define

$W_{a}^{+}:=\{w:$ a cadlag function from $[0, \zeta(w)]$ to $E$ for some $\zeta(w) \in(0, \infty)$ with $w(t) \in E_{0}$ for $t \in(0, \zeta(w))$ and $\left.w(0)=w(\zeta(w))=w(\zeta(w)-)=a\right\}$, $W_{a}^{-}:=W_{a} \backslash W_{a}^{+}$. 
The excursion law $\mathbf{n}$ will be considered to be a measure on $W_{a}$ defined by (5.23). Let us add an extra point $\partial$ to $W_{a}$ which represents a specific path constantly equal to $\Delta$. Fix a non-negative constant $\delta_{0}$ and we assign a point mass $\delta_{0}$ to $\{\partial\}$ and extend the measure $\mathbf{n}$ on $W_{a}$ to a measure $\overline{\mathbf{n}}$ on $W_{a} \cup\{\partial\}$ by

$$
\overline{\mathbf{n}}(\Lambda)=\left\{\begin{array}{lll}
\mathbf{n}(\Lambda) & \text { if } & \Lambda \subset W_{a} \\
\mathbf{n}\left(\Lambda \cap W_{a}\right)+\delta_{0} & \text { if } & \partial \in \Lambda
\end{array}\right.
$$

for $\Lambda \subset W_{a} \cup\{\partial\}$. The restrictions of $\overline{\mathbf{n}}$ to $W_{a}^{+}$and $W_{a}^{-} \cup\{\partial\}$ are denoted by $\mathbf{n}^{+}$and $\overline{\mathbf{n}}^{-}$, respectively.

Let $\mathbf{p}=\left\{\mathbf{p}_{s}: s \in \mathcal{D}_{\mathbf{p}}\right\}$ be a Poisson point process on $W_{a} \cup\{\partial\}$ with characteristic measure $\overline{\mathbf{n}}$ defined on an appropriate probability space $\left(\Omega_{a}, \mathbf{P}\right)$. We then let $\mathbf{p}^{+}$and $\mathbf{p}^{-}$be the point processes obtained from $\mathbf{p}$ by restricting to $W_{a}^{+}$and $W_{a}^{-} \cup\{\partial\}$ respectively, that is,

$\mathcal{D}_{\mathbf{p}^{+}}=\left\{s \in \mathcal{D}_{\mathbf{p}}: \mathbf{p}_{s} \in W_{a}^{+}\right\} \quad$ and $\quad \mathcal{D}_{\mathbf{p}^{-}}=\left\{s \in \mathcal{D}_{\mathbf{p}}: \mathbf{p}_{s} \in W_{a}^{-} \cup\{\partial\}\right\}$.

Then $\left\{\mathbf{p}_{s}^{+}, s>0\right\},\left\{\mathbf{p}_{s}^{-}, s>0\right\}$ are mutually independent Poisson point processes on $W_{a}^{+}$and $W_{a}^{-} \cup\{\partial\}$ with characteristic measures $\mathbf{n}^{+}$and $\overline{\mathbf{n}}^{-}$, respectively. Clearly,

$$
\mathbf{p}_{s}=\mathbf{p}_{s}^{+}+\mathbf{p}_{s}^{-} .
$$

Recall that $\zeta\left(\mathbf{p}_{r}^{+}\right)$denotes the terminal time of the excursion $\mathbf{p}_{r}^{+}$. We define

$$
J(s):=\sum_{r \leq s} \zeta\left(\mathbf{p}_{r}^{+}\right) \quad \text { for } s>0 \quad \text { and } \quad J(0):=0 .
$$

Lemma 5.6 (i) $J(s)<\infty$ a.s. for $s>0$.

(ii) $\{J(s)\}_{s \geq 0}$ is a subordinator with

$$
\mathbf{E}\left[e^{-\alpha J(s)}\right]=\exp \left(-\alpha\left(\widehat{u}_{\alpha}, \varphi\right) s\right) .
$$

Proof. (i) We write $J(s)$ as $J(s)=I+I I$ with

$$
I:=\sum_{r \leq s, \zeta\left(\mathbf{p}_{r}^{+}\right) \leq 1} \zeta\left(\mathbf{p}_{r}^{+}\right) \quad \text { and } \quad I I:=\sum_{r \leq s, \zeta\left(\mathbf{p}_{r}^{+}\right)>1} \zeta\left(\mathbf{p}_{r}^{+}\right) .
$$

Since $\mathbf{n}^{+}(\zeta>1) \leq \mu_{1}\left(E_{0}\right)<\infty$ by $(5.10), r$ in the sum $I I$ is finite a.s. and hence $I I<\infty$ a.s. On the other hand,

$$
\begin{aligned}
\mathbf{E}(I) & =s \mathbf{n}^{+}(\zeta ; \zeta \leq 1) \leq s \mathbf{n}^{+}(\zeta \wedge 1) \\
& =s \mathbf{n}^{+}\left\{\int_{0}^{1} 1_{(0, \zeta)}(t) d t\right\}=s \int_{0}^{1} \mathbf{n}^{+}(\zeta>t) d t \leq s \int_{0}^{1} \mu_{t}\left(E_{0}\right) d t,
\end{aligned}
$$

which is finite by (5.10). Hence $I<\infty$ a.s.

(ii) This can be shown exactly in the same way as that for (4.18) in the proof of Theorem 4.2 by using the identity (5.9). 
In view of Lemma 5.4 and Lemma 5.6, by subtracting a $\mathbf{P}$-negligible set from $\Omega_{a}$ if necessary, we may and do assume that the next three properties hold for every $\omega \in \Omega_{a}$ :

$$
\begin{gathered}
J(s)<\infty \quad \text { for every } s>0, \\
\lim _{s \rightarrow \infty} J(s)=\infty,
\end{gathered}
$$

and, for any finite interval $I \subset(0, \infty)$ and any neighborhood $U$ of $a$ in $E$,

$$
\left\{s \in I: \tau_{U}\left(\mathbf{p}_{s}^{+}\right)<\zeta\left(\mathbf{p}_{s}^{+}\right)\right\} \text {is a finite set. }
$$

Let $T$ be the first time of occurrence of the point process $\left\{\mathbf{p}_{s}^{-}, s>0\right\}$, namely,

$$
T=\inf \left\{s>0: s \in \mathcal{D}_{\mathbf{p}^{-}}\right\} .
$$

Since by Lemma 5.5

$$
\overline{\mathbf{n}}^{-}\left(W_{a}^{-} \cup\{\partial\}\right)=\mathbf{n}\left(W_{a}^{-}\right)+\delta_{0}=L^{0}\left(\widehat{\varphi} \cdot m_{0}, 1-\varphi\right)+\delta_{0}<\infty,
$$

we see that $T$ and $\mathbf{p}_{T}^{-}$are independent and

$\mathbf{P}(T>t)=e^{-\left(L\left(\widehat{\varphi} \cdot m_{0}, 1-\varphi\right)+\delta_{0}\right) t} \quad$ and $\quad \mathbf{p}_{T}^{-} \stackrel{\text { dist }}{=}\left(L\left(\widehat{\varphi} \cdot m_{0}, 1-\varphi\right)+\delta_{0}\right)^{-1} \overline{\mathbf{n}}^{-}$.

We are now in a position to produce a new process $X=\left\{X_{t}, t \geq 0\right\}$ out of the point processes of excursions $\mathbf{p}^{ \pm}$.

(i) For $0 \leq t<J(T-)$, there is an $s \geq 0$ such that

$$
J(s-) \leq t \leq J(s) .
$$

We define

$$
X_{t}^{a}:= \begin{cases}\mathbf{p}_{s}^{+}(t-J(s-)) & \text { if } J(s)-J(s-)>0 \\ a & \text { if } J(s)-J(s-)=0 .\end{cases}
$$

It is easy to see that $X^{a}$ is well-defined.

(ii) If $\mathbf{p}_{T}^{-} \in W_{a}^{-}$, then we define

$$
\zeta_{\omega}:=J(T-)+\zeta\left(\mathbf{p}_{T}^{-}\right) \quad \text { and } \quad X_{t}^{a}:=\mathbf{p}_{T}^{-}(t-J(T-)) \quad \text { for } \quad J(T-) \leq t<\zeta_{\omega} .
$$

(iii) If $\mathbf{p}_{T}^{-}=\partial$, then we define

$$
\zeta_{\omega}:=J(T-) .
$$

In this way, the $E$-valued path

$$
\left\{X_{t}^{a}, 0 \leq t<\zeta_{\omega}\right\}
$$

is well-defined and enjoys the following properties: 
$X_{0}^{a}=a$, is cadlag in $t \in\left[0, \zeta_{\omega}\right)$ and continuous when $X_{t}^{a}=a$, and $X_{\zeta_{\omega}-}^{a} \in\{a, \Delta\}$ whenever $\zeta_{\omega}<\infty$.

The second property is a consequence of (5.30). If $\mathbf{p}_{T}^{-} \in W_{a}^{-}$and $\zeta_{\omega}<\infty$, then $X_{\zeta_{\omega}-}^{a}=\Delta$. If $T<\infty, \mathbf{p}_{T}^{-}=\partial$, then $T \notin \mathcal{D}_{\mathbf{p}^{+}}$and hence by (5.35), we have $X_{\zeta_{\omega-}}^{a}=X_{J(T-)-}^{a}=a$. Thus the third property holds.

For this process $X^{a}=\left\{X_{t}^{a}, 0 \leq t<\zeta_{\omega}, \mathbf{P}\right\}$, let us put

$$
G_{\alpha} f(a)=\mathbf{E}\left[\int_{0}^{\zeta_{\omega}} e^{-\alpha t} f\left(X_{t}^{a}\right) d t\right], \quad \alpha>0, f \in \mathcal{B}(E) .
$$

Similarly we assign a non-negative mass $\widehat{\delta}_{0}$ to the death path $\partial$ and extend the measure $\widehat{\mathbf{n}}$ on $W_{a}$ to a measure $\overline{\hat{\mathbf{n}}}$ on $W_{a} \cup\{\partial\}$. By making use of the Poisson point process $\widehat{\mathbf{p}}$ on $W_{a} \cup\{\partial\}$ with the characteristic measure $\overline{\hat{\mathbf{n}}}$ on a certain probability space $\left(\widehat{\Omega}_{a}, \widehat{\mathbf{P}}\right)$, we can construct a cadlag process $\left\{\widehat{X}_{t}^{a}, 0 \leq t<\right.$ $\left.\widehat{\zeta}_{\hat{\omega}}, \widehat{\mathbf{P}}\right)$ on $E$ quite analogously. The corresponding quantity to (5.37) is denoted by $\widehat{G}_{\alpha} f(a)$. We can then obtain the first identity of the next proposition exactly in the same way as in the proof of Theorem 4.2 using (5.9), Lemma 5.6 and (5.32). An analogous consideration gives the second identity.

Proposition 5.7 For $\alpha>0$ and $f \in \mathcal{B}(E)$, it holds that

$$
\begin{aligned}
G_{\alpha} f(a) & =\frac{\left(\widehat{u}_{\alpha}, f\right)}{\alpha\left(\widehat{u}_{\alpha}, \varphi\right)+L^{(0)}\left(\widehat{\varphi} \cdot m_{0}, 1-\varphi\right)+\delta_{0}} . \\
\widehat{G}_{\alpha} f(a) & =\frac{\left(u_{\alpha}, f\right)}{\alpha\left(u_{\alpha}, \widehat{\varphi}\right)+\widehat{L}^{0}\left(\varphi \cdot m_{0}, 1-\widehat{\varphi}\right)+\widehat{\delta}_{0}} .
\end{aligned}
$$

For $\alpha>0$ and $f \in \mathcal{B}(E)$, define

$$
\begin{array}{ll}
G_{\alpha} f(x):=G_{\alpha}^{0} f(x)+G_{\alpha} f(a) u_{\alpha}(x) & \text { for } x \in E_{0}, \\
\widehat{G}_{\alpha} f(x):=\widehat{G}_{\alpha}^{0} f(x)+\widehat{G}_{\alpha} f(a) \widehat{u}_{\alpha}(x) & \text { for } x \in E_{0} .
\end{array}
$$

Lemma $5.8\left\{G_{\alpha}, \alpha>0\right\}$ and $\left\{\widehat{G}_{\alpha}, \alpha>0\right\}$ are sub-Markovian resolvents on $E$. They are in weak duality with respect to $m$ if and only if

$$
L^{(0)}\left(\widehat{\varphi} \cdot m_{0}, 1-\varphi\right)+\delta_{0}=\widehat{L}^{(0)}\left(\varphi \cdot m_{0}, 1-\widehat{\varphi}\right)+\widehat{\delta}_{0} .
$$

Proof. By making use of the resolvent equations for $G_{\alpha}^{0}, \widehat{G}_{\alpha}^{0}$, their weak duality with respect to $m_{0}$ and the equations

$$
\begin{aligned}
& u_{\alpha}(x)-u_{\beta}(x)+(\alpha-\beta) G_{\alpha}^{0} u_{\beta}(x)=0, \quad \alpha, \beta>0, x \in E_{0}, \\
& \widehat{u}_{\alpha}(x)-\widehat{u}_{\beta}(x)+(\alpha-\beta) \widehat{G}_{\alpha}^{0} \widehat{u}_{\beta}(x)=0, \quad \alpha, \beta>0, x \in E_{0},
\end{aligned}
$$

we can easily check the resolvent equations 


$$
\begin{aligned}
& G_{\alpha} f(x)-G_{\beta} f(x)+(\alpha-\beta) G_{\alpha} G_{\beta} f(x)=0, \quad x \in E, \\
& \widehat{G}_{\alpha} f(x)-\widehat{G}_{\beta} f(x)+(\alpha-\beta) \widehat{G}_{\alpha} \widehat{G}_{\beta} f(x)=0, \quad x \in E .
\end{aligned}
$$

Moreover we get as in [16, Lemma 2.1] that

$$
\begin{aligned}
\alpha G_{\alpha} 1(x) & =\alpha G_{\alpha}^{0} 1(x)+u_{\alpha}(x) \frac{\alpha\left(\widehat{u}_{\alpha}, \varphi\right)+\alpha\left(\widehat{u}_{\alpha}, 1-\varphi\right)}{\alpha\left(\widehat{u}_{\alpha}, \varphi\right)+L\left(\widehat{\varphi} \cdot m_{0}, 1-\varphi\right)+\delta_{0}} \\
& \leq 1-u_{\alpha}(x)+u_{\alpha}(x)=1, \quad x \in E_{0},
\end{aligned}
$$

and similarly, $\alpha G_{\alpha} 1(a) \leq 1$.

The $m$-weak duality

$$
\int_{E} \widehat{G}_{\alpha} f(x) g(x) m(d x)=\int_{E} f(x) G_{\alpha} g(x) m(d x), \quad f, g \in \mathcal{B}^{+}(E),
$$

holds if and only if the denominators of the right hand sides of (5.38) and (5.39) coincide. Since $\left(\widehat{u}_{\alpha}, \varphi\right)=\left(u_{\alpha}, \widehat{\varphi}\right)$ by the above equations for $u_{\alpha}, \widehat{u}_{\alpha}$, we get the last conclusion.

\subsection{Regularity of resolvent along the path of $X^{a}$}

Let $\left\{U_{n}\right\}$ be a decreasing sequence of open neighborhoods of the point $a$ in $E$ such that $U_{n} \supset \bar{U}_{n+1}$ and $\bigcap_{n=1}^{\infty} U_{n}=\{a\}$. For $\alpha>0$ and $0<\rho<1$, let

$$
A=A_{\alpha, \rho}:=\left\{x \in E_{0}: u_{\alpha}(x)<\rho\right\} .
$$

We then define

$$
\sigma_{n}:=\inf \left\{t>0: X_{t}^{0} \in U_{n} \cap E_{0}\right\}, \quad \tau_{n}:=\inf \left\{t>0: X_{t}^{0} \in U_{n} \cap A\right\},
$$

and $\sigma:=\lim _{n \rightarrow \infty} \sigma_{n}$, with the convention that $\inf \emptyset=\infty$. The stopping time $\sigma$ may be called the approaching time to $a$ of $X^{0}$.

The next lemma can be proved exactly in the same way as the proof of [16, Lemma 4.7].

Lemma 5.9 For any $\alpha>0, \rho \in(0,1)$ and $x \in E_{0}$,

$$
\lim _{n \rightarrow \infty} \mathbf{P}_{x}^{0}\left\{\tau_{n}<\sigma<\infty\right\}=0 .
$$

Lemma 5.10 The following are ture.

(i) For any $x \in E_{0}, \mathbf{P}_{x}^{0}$-a.s. on $\{\sigma<\infty\}$,

$$
\lim _{t \uparrow \sigma} u_{\alpha}\left(X_{t}^{0}\right)=1 \quad \text { for every } \alpha>0 .
$$


(ii) $\mathbf{n}\left(\Lambda \cap W_{a}^{+}\right)=0$ where

$$
\Lambda=\left\{w \in W_{a}: \exists \alpha>0, \liminf _{t \uparrow \zeta} u_{\alpha}(w(t))<1\right\} .
$$

(iii) $\mathbf{n}(\widehat{\Lambda})=0$ where

$$
\widehat{\Lambda}=\left\{w \in W_{a}: \exists \alpha>0, \liminf _{t \downarrow 0} \widehat{u}_{\alpha}(w(t))<1\right\} .
$$

Proof. Let $0<\rho<1$. If $\sigma<\infty$ and if $\underline{\lim }_{t \uparrow \sigma} u_{\alpha}\left(X_{t}^{0}\right)<\rho$, then for any small $\epsilon>0$ there exists $t \in(\sigma-\epsilon, \sigma)$ such that $u_{\alpha}\left(X_{t}^{0}\right)<\rho$, and so $\tau_{n}<\sigma$ for all $n$. Therefore by the preceding lemma

$$
\mathbf{P}_{x}^{0}\left(\liminf _{t \uparrow \sigma} u_{\alpha}\left(X_{t}^{0}\right)<\rho, \sigma<\infty\right)=0 .
$$

Since $u_{\alpha}$ is decreasing in $\alpha$ and $\rho$ can be taken arbitrarily close to 1 , we obtain (5.46).

(ii) follows from (i) as

$$
\begin{aligned}
& \mathbf{n}\left(\Lambda \cap W_{a}^{+}\right)=\lim _{\epsilon \downarrow 0} \mathbf{n}\left(\Lambda \cap W_{a}^{+} \cap\{\epsilon<\zeta\}\right) \\
= & \lim _{\epsilon \downarrow 0} \int_{E_{0}} \mu_{\epsilon}(d x) \mathbf{P}_{x}^{0}\left(\liminf _{t \uparrow \sigma} u_{\alpha}\left(X_{t}^{0}\right)<1, \sigma<\infty \text { for every } \alpha>0\right)=0 .
\end{aligned}
$$

(iii). Part (ii) combined with Lemma 5.2 and a similar reasoning as in the proof of Lemma 5.3 leads us to

$$
\mathbf{n}\left(\widehat{\Lambda} \cap W_{a}^{+}\right)=\widehat{\mathbf{n}}\left(\{\widehat{w} \in \widehat{\Lambda}\} \cap W_{a}^{+}\right)=0,
$$

and also $\mathbf{n}(\widehat{\Lambda})=0$.

Denote by $Q^{+}$the set of all positive rational number and by $C_{b}(E)$ the space of all bounded continuous functions on $E$. Let us fix an arbitrary countable subfamily $\mathbf{L}$ of $C_{b}(E)$. We extend functions $u_{\alpha}(x)$ and $G_{\alpha}^{0} f(x)$ for $f \in C_{b}(E)$ to be functions on $E$ by setting $u_{\alpha}(a)=1$ and $G_{\alpha}^{0} f(a)=0$ respectively. Functions $\widehat{u}_{\alpha}$ and $\widehat{G}_{\alpha}^{0} f$ are similarly extended to $E$.

As $u_{\alpha}$ and $G_{\alpha}^{0} f$ for a non-negative $f \in C_{b}(E)$ are $\alpha$-excessive with respect to the process $X^{0}$, it is well-known (cf. [2]) that

$u_{\alpha}\left(X_{t}^{0}\right), G_{\alpha}^{0} f\left(X_{t}^{0}\right)$ are right continuous in $t \in[0, \zeta) \quad \mathbf{P}_{x}^{0}-$ a.s. $\quad x \in E_{0}$.

Suppose that $X^{0}$ is $m_{0}$-symmetric: $X^{0}=\widehat{X}^{0}$. Then $u_{\alpha}=\widehat{u}_{\alpha}$ and hence by Lemma 5.10

$$
\mathbf{n}\left(\liminf _{t \downarrow 0} u_{\alpha}(w(t))<1\right)=0 .
$$


On account of (5.47) and the inequality $a G_{\alpha}^{0} 1(x) \leq 1-u_{\alpha}(x), x \in E$, after subtracting a suitable $\mathbf{n}$-negligible set from $W_{a}$ if necessary, we may and do assume that, for any $f \in \mathbf{L}, \alpha \in Q^{+}$,

$u_{\alpha}(w(t))$ and $G_{\alpha}^{0} f(w(t))$ are right continuous in $t \in[0, \zeta)$ for $w \in W_{a}$, $u_{\alpha}(w(\zeta-))=1, G_{\alpha}^{0} f(w(\zeta-))=0$, for $w \in W_{a}^{+}$.

When $X^{0}$ is non-symmetric, $u_{\alpha} \neq \widehat{u}_{\alpha}$ and the above argument does not work. However, since we have assumed in this non-symmetric case the condition (A.5), the above property (5.48) holds by Lemma 5.3.

Lemma 5.11 Let $0<\rho<1$ and set, for $\alpha>0$,

$$
\widetilde{W}_{\rho}=\left\{w \in W_{a}^{+}: \sup _{0 \leq t \leq \zeta}\left\{1-u_{\alpha}(w(t))\right\}>\rho\right\} .
$$

Then $\mathbf{n}^{+}\left(\widetilde{W}_{\rho}\right)<\infty$.

Proof. Define $\delta:=-\frac{1}{\alpha} \log \left(1-\frac{\rho}{2}\right)>0$. For any $x$ with $1-u_{\alpha}(x) \geq \rho$, we have

$$
\begin{aligned}
& \mathbf{P}_{x}^{0}(\sigma>\delta) \geq \mathbf{E}_{x}^{0}\left[1-e^{-\alpha \sigma} ; \sigma>\delta\right]=\mathbf{E}_{x}^{0}\left[1-e^{-\alpha \sigma}\right]-\mathbf{E}_{x}^{0}\left[1-e^{-\alpha \sigma} ; \sigma \leq \delta\right] \\
\geq & 1-u_{\alpha}(x)-\left(1-e^{-\alpha \delta}\right) \geq \rho-\left(1-e^{-\alpha \delta}\right)=\frac{\rho}{2} .
\end{aligned}
$$

Therefore if we define

$$
\tau:=\inf \left\{t>0: 1-u_{\alpha}(w(t))>\rho\right\},
$$

then for any neighborhood $U$ of $a$,

$$
\begin{aligned}
& \mathbf{n}^{+}\left(\widetilde{W}_{\rho}\right)=\mathbf{n}^{+}\left(\tau<\zeta^{0}\right)=\lim _{\epsilon \downarrow 0} \mathbf{n}^{+}\left(\epsilon<\tau<\zeta^{0}\right) \\
= & \lim _{\epsilon \downarrow 0} \int_{E_{0}} \mu_{\epsilon}(d x) \mathbf{P}_{x}^{0}\left(\tau<\zeta^{0}<\infty\right) \\
\leq & \liminf _{\epsilon \downarrow 0} \int_{E_{0}} \mu_{\epsilon}(d x) \mathbf{E}_{x}^{0}\left[\left(\frac{2}{\rho}\right) \mathbf{P}_{X_{\tau}^{0}}^{0}(\sigma>\delta) ; \tau<\zeta^{0}\right] \\
\leq & \frac{2}{\rho} \liminf _{\epsilon \downarrow 0} \int_{E_{0}} \mu_{\epsilon}(d x) \mathbf{P}_{x}^{0}\left(\sigma>\delta, \zeta^{0}<\infty\right) \\
\leq & \frac{2}{\rho} \lim _{\epsilon \downarrow 0} \int_{E_{0}} \mu_{\epsilon}(d x) \mathbf{P}_{x}^{0}\left(\zeta^{0}>\delta\right)+\frac{2}{\rho} \lim _{\epsilon \downarrow 0} \int_{E_{0}} \mu_{\epsilon}(d x) \mathbf{P}_{x}^{0}\left(\zeta^{0}<\infty, X_{\zeta^{0}-}=\Delta\right) \\
\leq & \frac{2}{\rho} \lim _{\epsilon \downarrow 0} \mu_{\epsilon+\delta}\left(E_{0}\right)+\frac{2}{\rho} \mathbf{n}\left(\tau_{U}<\zeta\right),
\end{aligned}
$$

which is finite in view of (5.10) and Lemma 5.4. 
In last subsection, we have constructed a process $X^{a}=\left\{X_{t}^{a}, t \in\left[0, \zeta_{\omega}\right)\right\}$ starting from $a$ out of the Poisson point processes $\mathbf{p}^{+}$and $\mathbf{p}^{-}$on $W_{a}^{+}$and $W_{a}^{-} \cup$ $\{\partial\}$ defined on a probability space $(\Omega, \mathbf{P})$, respectively. A process $\left\{\widehat{X}_{t}^{a}, t \in\right.$ $\left.\left[0, \widehat{\zeta}_{\widehat{\omega}}\right)\right\}$ can be constructed similarly.

Proposition 5.12 Let $v(x)=G_{\alpha} f$ with $f \in C_{b}(E)$ be defined by (5.38) and (5.40). Then $v\left(X_{t}^{a}\right)$ is right continuous in $t \in\left[0, \zeta_{\omega}\right)$ and is continuous when $X_{t}=a$ for every $f \in \mathbf{L}$ and every $\alpha \in Q^{+} \mathbf{P}$-a.s. An analogous property holds for $\widehat{X}^{a}$.

Proof. We already saw that the functions $u_{\alpha}$ and $G_{\alpha}^{0} f$ for $f \in \mathbf{L}, \alpha \in \mathbf{Q}^{+}$, have the property (5.48) along any sample point functions of $\mathbf{p}^{+}=\left\{\mathbf{p}_{s}^{+}, s>0\right\}$ and $\mathbf{p}^{-}=\left\{\mathbf{p}_{s}^{-}, s>0\right\}$. Moreover, by Lemma 5.11, after subtracting a suitable $\mathbf{P}$-negligible set from $\Omega$ if necessary, we can assume that, in addition to the properties (5.28), (5.29) and (5.30), $\mathbf{p}^{+}$satisfies the following property for every sample point $\omega \in \Omega$ : for any finite interval $I \subset(0, \infty)$ and for any $\rho \in(0,1)$,

$$
\left\{s \in I: \sup _{0 \leq t \leq \zeta\left(\mathbf{p}_{s}^{+}\right)}\left(1-u_{\alpha}\left(\mathbf{p}_{s}^{+}(t)\right)\right)>\rho\right\} \text { is a finite set. }
$$

Combining this with the inequality $\alpha G_{\alpha}^{0} 1(x) \leq 1-u_{\alpha}(x), x \in E$, it is not hard to see that $u_{\alpha}\left(X_{t}^{a}\right), G_{\alpha}^{0} f\left(X_{t}^{a}\right)$ and hence $v\left(X_{t}^{a}\right)$ enjoy the properties in the statement of the proposition.

\subsection{Constructing a standard process $X$ on $E_{0} \cup\{a\}$}

Combining the given standard process $X^{0}$ on $E_{0}$ with the process $X^{a}$ constructed and studied in the last two subsections, we can now construct a right process $X$ on $E:=E_{0} \cup\{a\}$ whose resolvent coincides with $\left\{G_{\alpha}, \alpha>0\right\}$ defined by (5.38) and (5.40). We will only do the construction of $X$. But obviously the analogous procedure allows us to construct out of $\widehat{X}^{0}$ a right process $\widehat{X}$ on $E$ with resolvent given by (5.39) and(5.41), and these two right processes on $E$ are in weak duality with respect to $m$ if and only if their killing rates $\delta_{0}$ and $\widehat{\delta}_{0}$ at $a$ satisfy the relation (4.10).

With the preparations made in the last subsections, we can now just follow the corresponding arguments in $[16, \S 4]$ without any essential change to construct the desired process $X$ on $E$.

First, using the approaching time $\sigma$ to $a$ of $X^{0}$ defined in the beginning of the last subsection, we define $P_{t} f(x)$ for $t>0, x \in E, f \in \mathcal{B}(E)$, as follows:

$$
\begin{aligned}
& P_{t} f(a):=\mathbf{E}\left(f\left(X_{t}^{a}\right) ; t<\zeta_{\omega}\right), \\
& P_{t} f(x):=P_{t}^{0} f(x)+\mathbf{E}_{x}^{0}\left[P_{t-\sigma} f(a) ; \sigma \leq t\right] \quad \text { for } x \in E_{0} .
\end{aligned}
$$


Evidently the Laplace transform of $P_{t}$ equals the resolvent $G_{\alpha}$ in view of (5.37) and (5.40) and we can see exactly in the same way as the proof of $[16$, Lemma 4.10] that $\left\{P_{t}, t \geq 0\right\}$ is a sub-Markovian transition semigroup on $E$ :

$$
P_{t+s}=P_{t} P_{s} \quad \text { with } \quad P_{t} 1 \leq 1 \quad \text { for } t, s>0 .
$$

Proposition 5.13 (i) $X^{a}=\left\{X_{t}^{a}, 0 \leq t<\zeta_{\omega}, \mathbf{P}\right\}$ is a Markov process on $E$ starting from a with transition semigroup $\left\{P_{t}, t>0\right\}$.

(ii) $\mathbf{P}\left(\sigma_{a}=0, \tau_{a}=0\right)=1$, where $\sigma_{a}=\inf \left\{t>0: X_{t}^{a}=a\right\}$ and $\tau_{a}=\inf \{t>$ $\left.0: X_{t}^{a} \in E_{0}\right\}$.

Proof. The proof of [16, Proposition 4.4] still works to obtain the first assertion (i). The only places to be modified in the proof are to replace $L\left(m_{0}, \psi\right)$ appearing there with $L^{0}\left(\widehat{\varphi} \cdot m_{0}, 1-\varphi\right)+\delta_{0}$ in the present case.

The second assertion (ii) follows from (i) and Proposition 5.12 just as the proof of [16, Lemma 4.12].

In $\S 5.1$, we have started with a standard process

$$
X^{0}=\left\{X_{t}^{0}, 0 \leq t<\zeta^{0}, \mathbf{P}_{x}^{0}, x \in E_{0}\right\}
$$

on $E_{0}$, where $\mathbf{P}_{x}^{0}, x \in E_{0}$, are probability measures on a certain sample space, say $\Omega^{0}$.

In $\S 5.2$, we have constructed a cadlag process

$$
X^{a}=\left\{X_{t}^{a}\left(\omega^{\prime}\right), 0 \leq t<\zeta_{\omega^{\prime}}, \mathbf{P}\right\}
$$

on $E$ starting from $a$ by piecing together excursions away from $a$, where $\mathbf{P}$ is a probability measure on another sample space, say $\Omega^{\prime}$, to define the Poisson point process with value in $\left(W_{a} \cup\{\partial\}, \overline{\mathbf{n}}\right)$.

For convenience, we assume that $\Omega^{0}$ contains an extra path $\eta$ with $\mathbf{P}_{x}^{0}(\{\eta\})=0$ for every $x \in E_{0}$, and we set $\mathbf{P}_{a}^{0}=\delta_{\eta}, \eta$ representing the constant path taking value $a$ identically.

We now define

$$
\Omega=\Omega^{0} \times \Omega^{\prime}, \quad \mathbf{P}_{x}=\mathbf{P}_{x}^{0} \times \mathbf{P} \quad \text { for } x \in E .
$$

Note that $\zeta^{0}\left(\omega^{0}\right) \leq \sigma\left(\omega^{0}\right)$ and $\zeta^{0}\left(\omega^{0}\right)=\sigma\left(\omega^{0}\right)$ when $\sigma\left(\omega^{0}\right)<\infty$. For $\omega=$ $\left(\omega^{0}, \omega^{\prime}\right) \in \Omega$, let us define $X_{t}=X_{t}(\omega)$ as follows:

(1) When $\omega^{0} \in \Omega^{0} \backslash\{\eta\}$,

$$
X_{t}(\omega)= \begin{cases}X_{t}^{0}\left(\omega^{0}\right) & 0 \leq t<\zeta^{0}\left(\omega^{0}\right) \leq \sigma\left(\omega^{0}\right) \leq \infty \\ X_{t-\sigma\left(\omega^{0}\right)}^{a}\left(\omega^{\prime}\right) & \sigma\left(\omega^{0}\right) \leq t<\sigma\left(\omega^{0}\right)+\zeta_{\omega^{\prime}}, \text { if } \sigma\left(\omega^{0}\right)<\infty .\end{cases}
$$

(2) When $\omega^{0}=\eta$, 


$$
X_{t}(\omega)=X_{t}^{a}\left(\omega^{\prime}\right) \quad 0 \leq t<\zeta_{\omega^{\prime}} .
$$

The life time $\zeta(\omega)$ of $X_{t}(\omega)$ is defined by

$$
\zeta(\omega)= \begin{cases}\zeta^{0}\left(\omega^{0}\right) & \text { if } \sigma\left(\omega^{0}\right)=\infty \\ \sigma\left(\omega^{0}\right)+\zeta_{\omega^{\prime}} & \text { if } \sigma\left(\omega^{0}\right)<\infty\end{cases}
$$

Combining Proposition 5.13(i) with the Markov property of $\left\{X_{t}^{0}, t \geq\right.$ $\left.0, \mathbf{P}_{x}^{0}, x \in E_{0}\right\}$, we readily get as in [16, Lemma 4.13] the next lemma:

Lemma 5.14 $X=\left\{X_{t}, 0 \leq t<\zeta, \mathbf{P}_{x}, x \in E\right\}$ is a Markov process on $E$ with transition semigroup $\left\{P_{t}, t \geq 0\right\}$ defined by (5.50) and (5.51).

The resolvent $\left\{G_{\alpha}, \alpha>0\right\}$ of the Markov process $X$ is defined by

$$
G_{\alpha} f(x)=\mathbf{E}_{x}\left[\int_{0}^{\infty} e^{-\alpha t} f\left(X_{t}\right) d t\right], \quad x \in E, \alpha>0, f \in \mathcal{B}(E) .
$$

The resolvent of $X^{0}$ is denoted by $G_{\alpha}^{0}$.

Theorem 5.15 The process $X$ enjoys the following properties:

(i) $X$ is a right process on $E$. Its sample path $\left\{X_{t}, 0 \leq t<\zeta\right\}$ is cadlag on $[0, \infty)$, continuous when $X_{t}=a$ and satisfies

$$
X_{\zeta-} \in\{a, \Delta\} \quad \text { when } \quad \zeta<\infty .
$$

(ii) The point a is regular for itself with respect to $X$ in the sense that for the hitting time $\sigma_{a}=\inf \left\{t>0: X_{t}=a\right\}$

$$
\mathbf{P}_{a}\left(\sigma_{a}=0\right)=1 .
$$

(iii) $X^{0}$ is identical in law with the subprocess of $X$ killed upon hitting a.

(iv) The resolvent $G_{\alpha} f$ admits the expression (5.38) and (5.40) for $f \in \mathcal{B}(E)$.

(v) If $X^{0}$ is a diffusion on $E_{0}$, then $X$ is a diffusion on $E$.

Proof. (iv) follows from Lemma 5.14 and a statement next to (5.51).

(i). On account of (A.1), we may assume that

$$
\begin{aligned}
& X_{t}^{0}\left(\omega^{0}\right) \text { is cadlag in } t \in\left[0, \zeta^{0}\left(\omega^{0}\right)\right) \text { and } \\
& X_{\zeta^{0}\left(\omega^{0}\right)_{-}}^{0}\left(\omega^{0}\right) \in\{a \cup \Delta\} \text { when } \zeta^{0}\left(\omega^{0}\right)<\infty,
\end{aligned}
$$

for every $\omega^{0} \in \Omega^{0}$. We have already chosen $\Omega^{\prime}$ in a way that $\left\{X_{t}^{a}\left(\omega^{\prime}\right), 0 \leq\right.$ $\left.t<\zeta_{\omega^{\prime}}\right\}$ has the property (5.36). Hence the sample path $t \mapsto X_{t}(\omega)$ has the stated property in (i).

Take a countable linear subspace $\mathbf{L}$ of $C_{b}(E)$ such that, for any open set $G \subset E$, there exist functions $f_{n} \in \mathbf{L}$ increasing to $I_{G}$. We then see from the 
expression (5.40) of $G_{\alpha} f,(5.47)$ and Proposition 5.12 that, for any $v=G_{\alpha} f$ with $f \in \mathbf{L}, \alpha \in Q^{+}$,

$$
v\left(X_{t}\right) \text { is right continuous in } t \in[0, \zeta) \quad \mathbf{P}_{x^{-}} \text {a.s. for } x \in E .
$$

Therefore $X$ is strong Markov by [2, p41].

(ii) follows from Proposition 5.13(ii).

(iii) and (v) are also evident from the construction of $X$.

The right process $X$ in the above theorem becomes a standard process if either condition (A.1) or (A.4) is replaced by the following stronger counterpart, respectively:

(A.1) ${ }^{\prime} \quad X^{0}$ and $\widehat{X}^{0}$ are standard processes on $E_{0}$ in weak duality with respect to $m$ and

$$
\text { every semipolar set is } m \text {-polar for } X^{0} \text {. }
$$

(A.4)' For any $\alpha>0, u_{\alpha}, \widehat{u}_{\alpha} \in C_{b}\left(E_{0}\right)$ and

$$
G_{\alpha}^{0}\left(C_{b}\left(E_{0}\right)\right) \subset C_{b}\left(E_{0}\right), \widehat{G}_{\alpha}^{0}\left(C_{b}\left(E_{0}\right)\right) \subset C_{b}\left(E_{0}\right) .
$$

We note that condition (5.57) is automatically satisfied if $X^{0}$ is $m$ symmetric or more generally if the Dirichlet form of $X^{0}$ on $L^{2}\left(E_{0} ; m_{0}\right)$ is sectorial (cf.[4]). (A.4)' implies (A.4) as we noted right after the statement of the latter. Recall that a right process is called a standard process if it is quasi-left continuous up to the life time.

Theorem 5.16 (i) Suppose that the standard processes $X^{0}$ and $\widehat{X}^{0}$ on $E_{0}$ satisfy (A.1), (A.2), (A.3), (A.4)' and additionally (A.5) in non-symmetric case and (A.6) in non-diffusion case. Then the right process $X$ on $E$ in Theorem 5.15 is quasi-left continuous up to the life time.

(ii) Suppose that the standard processes $X^{0}$ and $\widehat{X}^{0}$ on $E_{0}$ satisfy (A.1)', (A.2), (A.3), (A.4) and additionally (A.5) in non-symmetric case and (A.6) in non-diffusion case. Then the right process $X$ on $E$ in Theorem 5.15 is quasi-left continuous up to the life time for $X$-q.e. starting point $x \in E$.

Proof. (i) If condition (A.4)' is satisfied, then along any cadlag path of $X^{0}$, we trivially have

$$
\lim _{s \uparrow t} u_{\alpha}\left(X_{s}^{0}\right)=u_{\alpha}\left(X_{t-}^{0}\right) \quad \text { and } \quad \lim _{s \uparrow t} G_{\alpha}^{0} f\left(X_{s}^{0}\right)=G_{\alpha}^{0}\left(X_{t-}\right) \quad \text { for } t \in\left(0, \zeta^{0}\right) \text {, }
$$

for any $\alpha>0$ and $f \in C_{b}\left(E_{0}\right)$. Combining this with Lemma 5.10(i) and Lemma 5.11, we easily see as in the proofs of Proposition 5.12 and Theorem 5.15(i) that 


$$
\lim _{s \uparrow t} G_{\alpha} f\left(X_{s}\right)=G_{\alpha} f\left(X_{t-}\right), \quad t \in(0, \zeta), \quad \mathbf{P}_{x^{-}} \text {a.s. }
$$

for any $x \in E$ and for any $\alpha>0, f \in C_{b}(E)$, from which the quasi-left continuity of $X$ follows.

(ii) Here we use the terminologies adopted in [5]. From condition (A.1)', we can deduce as in [5, Lemma 2.2] that (5.58) holds $\mathbf{P}_{x}^{0}$-a.s. for $X^{0}$-q.e. $x \in E_{0}$ for each $\alpha>0$ and each $f \in C_{b}\left(E_{0}\right)$. In particular, there exists a Borel set $B \subset E_{0}$ with $m(B)=0$ such that $E_{0} \backslash B$ is $X^{0}$-invariant and (5.58) holds

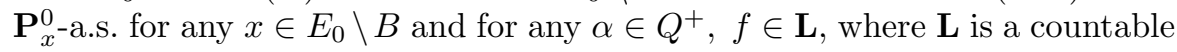
subfamily of $C_{b}\left(E_{0}\right)$.

Let us observe that the set $E \backslash B$ is invariant for $X$ of Theorem 5.15. Since the restriction of $X^{0}$ to the Lusin space $E_{0} \backslash B$ is a standard process again, the entrance law $\left\{\mu_{t}, t>0\right\}$ uniquely characterized by the equation (5.7) is carried by $E_{0} \backslash B$ for every $t>0$ and accordingly the excursion law $\mathbf{n}$ of Proposition 5.1 is carried by the path space (5.23) with $E, E_{0}$ being replaced by $E \backslash B, E_{0} \backslash B$ respectively. Hence $E \backslash B$ is $X$-invariant by the construction of $X$.

Now we can see by the same reasoning as in the proof of (i) that (5.59) holds for any $x \in E \backslash B$ and for any $\alpha \in Q^{+}, f \in \mathbf{L}$. Taking $\mathbf{L}$ as in the proof of Theorem 5.15(i), we conclude that $X$ is quasi-left continuous for every starting point $x \in E \backslash B$.

To formulate the last theorem in this section, we need the following stronger variant (A.2)' of the condition of (A.2):

(A.2)' For every $x \in E_{0}$,

$$
\begin{array}{ll}
\mathbf{P}_{x}^{0}\left(\zeta^{0}<\infty, X_{\zeta^{0}-}^{0}=a\right)>0, & \mathbf{P}_{x}^{0}\left(X_{\zeta^{0}-}^{0} \in\{a, \Delta\}\right)=1, \\
\widehat{\mathbf{P}}_{x}^{0}\left(\widehat{\zeta}^{0}<\infty, \widehat{X}_{\widehat{\zeta}^{0}-}^{0}=a\right)>0, & \widehat{\mathbf{P}}_{x}^{0}\left(\widehat{X}_{\widehat{\zeta}^{0}-}^{0} \in\{a, \Delta\}\right)=1 .
\end{array}
$$

Theorem 5.17 We assume that $m_{0}\left(U \cap E_{0}\right)<\infty$ for some neighborhood $U$ of a in E. Suppose that the pair of standard processes $X^{0}$ and $\widehat{X}^{0}$ on $E_{0}$ satisfy the conditions (A.1), (A.2)', (A.4) and additionally (A.5) in non-symmetric case and (A.6) in non-diffusion case. Then the integrability condition (A.3) is fulfilled by $X^{0}$ and $\widehat{X}^{0}$.

Proof. Note that the condition (A.3) holds if $m_{0}\left(E_{0}\right)<\infty$. When $m_{0}\left(E_{0}\right)=$ $\infty$, let $\gamma(x)$ be a continuous function on $E_{0}$ such that $0<\gamma(x) \leq 1$ on $E_{0}$, $\gamma(x)=1$ on $U \cap E_{0}$ and $\int_{E_{0}} \gamma(x) m_{0}(d x)<\infty$. Define for $t>0$,

$$
\tau_{t}:=\inf \left\{s>0: \int_{0}^{s} \gamma\left(X_{r}^{0}\right) d r>t\right\}
$$

and 


$$
\widehat{\tau}_{t}:=\inf \left\{s>0: \int_{0}^{s} \gamma\left(\widehat{X}_{r}^{0}\right) d r>t\right\} .
$$

Then the time changed processes $Y^{0}=\left\{Y_{t}^{0}:=X_{\tau_{t}}^{0}, t \geq 0\right\}$ and $\widehat{Y}^{0}=\left\{\widehat{Y}_{t}^{0}:=\right.$ $\left.\widehat{X}_{\widehat{\tau}_{t}}^{0}, t \geq 0\right\}$ are standard processes on $E_{0}$ satisfying (A.1) with respect to the finite measure $\mu_{0}=\gamma(x) m_{0}(d x)$. Clearly condition (A.3) holds for $Y^{0}$ and the reference measure $\mu_{0}$. Note that since $\gamma(x) \leq 1$, we have

$$
\tau_{t} \geq t \quad \text { and } \quad \widehat{\tau}_{t} \geq t \quad \text { for every } t \geq 0 .
$$

Let $G_{\alpha}^{Y^{0}}$ denote the 0-order resolvent of $Y^{0}$. It is easy to check that for any non-negative Borel function $f$ on $E_{0}, G^{Y^{0}} f=G^{0}(\gamma f)$. Therefore $Y^{0}$ and $\widehat{Y}^{0}$ inherit the conditions (A.2)', (A.4) and in non-symmetric case (A.5) from $X^{0}$ and $\widehat{X}^{0}$.

Let $(N, H)$ be a Lévy system of $X^{0}$. Since its defining formula (5.4) remains valid with the constant time $t$ being replaced by any stopping time, it follows from it and a time change that $Y^{0}$ has a Lévy system $\left(N, H^{Y^{0}}\right)$, where

$$
H_{t}^{Y^{0}}=H_{\tau_{t}} \quad \text { for every } t \geq 0 .
$$

According to [10, Theorem 6.2], the correspondence between PCAF and its Revuz measure is invariant under a strictly increasing time change. Therefore the Revuz measure of the PCAF of $H^{Y^{0}}$ with respect to the measure $\mu_{0}$ is the same as that $\mu_{H}$ of PCAF $H$ of $X^{0}$ with respect to the measure $m$. Hence $Y^{0}$ has the same jumping measure $J_{0}(d x, d y):=N(x, d y) \mu_{H}(d y)$ as that of $X^{0}$. The same applies to $\widehat{Y}^{0}$. Therefore $Y^{0}$ and $\widehat{Y}^{0}$ also inherit the condition (A.6) from $X^{0}$ and $\widehat{X}^{0}$.

Thus by Theorem 5.15 , there are duality preserving standard processes $Y$ and $\widehat{Y}$ on $E=E_{0} \cup\{a\}$ extending $Y^{0}$ and $\widehat{Y}^{0}$. Define for $t>0$,

$$
\sigma_{t}:=\inf \left\{s>0: \int_{0}^{s} \gamma\left(Y_{r}\right)^{-1} d r>t\right\}
$$

and

$$
\widehat{\sigma}_{t}:=\inf \left\{s>0: \int_{0}^{s} \gamma\left(\widehat{Y}_{r}\right)^{-1} d r>t\right\} .
$$

Then $X=\left\{X_{t}:=Y_{\sigma_{t}}, t \geq 0\right\}$ and $\widehat{X}=\left\{\widehat{X}_{t}:=\widehat{Y}_{\widehat{\sigma}_{t}}, t \geq 0\right\}$ is a pair of standard processes on $E$ in weak duality with respect to $m$. Clearly $X$ and $\widehat{X}$ extend $X^{0}$ and $\widehat{X}^{0}$, they spend zero Lebesgue amount of time at $\{a\}$, and for $X$ and $Y, a$ is a regular point for $\{a\}$. Therefore by Proposition 4.1(v), $X^{0}$ and $\widehat{X}^{0}$ must have the property (A.3).

Remark 3. In this section, we have assumed that $E$ is a locally compact separable metric space, $a$ is a non-isolated point of $E$ and $\Delta$ is added to $E$ 
as a one-point compactification. This assumption is used only to have (5.20) and (5.22).

The local compactness assumption on $E$ can be relaxed and be replaced by the following conditions. Let $E$ be a Lusin space and $a$ a non-isolated point of $E$ and $m_{0}$ be a $\sigma$-finite measure on $E_{0}:=E \backslash\{a\}$. Let $\Delta$ be a cemetery point added to $E$. Let $X^{0}$ and $\widehat{X}^{0}$ be Borel standard processes on $E_{0}$ with lifetimes $\zeta^{0}$ and $\widehat{\zeta}^{0}$, respectively.

We say $X_{\zeta^{0}-}^{0}=a$ if $\lim _{t \uparrow \zeta^{0}} X_{t}=a$ under the topology of $E$, and $X_{\zeta^{0}-}^{0}=\Delta$ if the $\operatorname{limit} \lim _{t \uparrow \zeta^{0}} X_{t}$ does not exist in the topology of $E$. The same applies to the process $\widehat{X}^{0}$.

Let $\left\{\mathcal{F}_{t}^{0}, t \geq 0\right\}$ be the minimal admissible completed $\sigma$-field generated by $X^{0}$. We assume $X^{0}$ and $\widehat{X}^{0}$ satisfy the conditions (A.1), (A.4)' and additionally (A.5) in non-symmetric case and (A.6) in non-diffusion case. We also assume, instead of (A.2), that

(A.2)" There is an open neighborhood $U_{1}$ of $a$ such that its closure $\overline{U_{1}}$ is compact in $E$. Further

$$
\begin{aligned}
& \zeta^{0} \text { is }\left\{\mathcal{F}_{t}^{0}\right\} \text {-predictable, } \varphi(x)>0 \text { on } E_{0} \text {, and } \liminf _{x \rightarrow a} \varphi(x)>0, \\
& \widehat{\zeta}^{0} \text { is } \widehat{\mathcal{F}}_{t}^{0} \text {-predictable, } \widehat{\varphi}(x)>0 \text { on } E_{0} \text {, and } \liminf _{x \rightarrow a} \widehat{\varphi}(x)>0
\end{aligned}
$$

where $\varphi$ is defined by (5.3) and $\widehat{\varphi}$ is defined analogously for $\widehat{X}^{0}$.

We claim that under the above assumptions, all the main results in this section, including Theorem 5.15, remain true. Note that the existence of an open neighborhood $U_{1}$ of $a$ with $\overline{U_{1}}$ being compact in $E$ guarantees the validity of (5.20). So it suffices to show that (5.22) holds almost surely under measure $\mathbf{n}$ for some neighborhood $U$ of $a$ under condition (5.60). As $c:=\liminf _{x \rightarrow a} \varphi(x)>0$ and $\varphi$ is lower semi-continuous by (A.4)', $U:=\left\{x \in E_{0}: \varphi(x)>c / 2\right\} \cup\{a\}$ is an open neighborhood of $a$. On the other hand, for $x \in E_{0}$, we have $\mathbf{P}_{x}^{0}$-a.s. on $\left\{t<\zeta^{0}\right\}$,

$$
\varphi\left(X_{t}^{0}\right)=\mathbf{E}_{x}\left[1_{\left\{\zeta^{0}<\infty \text { and } X_{\zeta^{0}-}^{0}=a\right\}} \mid \mathcal{F}_{t}^{0}\right] .
$$

As $\zeta^{0}$ is $\left\{\mathcal{F}_{t}^{0}\right\}$-predictable, it follows that

$$
\left.\lim _{t \uparrow \zeta^{0}} \varphi\left(X_{t}\right)=1_{\left\{\zeta^{0}<\infty\right.} \text { and } X_{\zeta^{0}-}^{0}=a\right\} \quad \mathbf{P}_{x^{-}} \text {a.s. for every } x \in E_{0} .
$$

Hence

$$
\left\{\zeta^{0}<\infty \text { and } X_{\zeta^{0}-}^{0}=\Delta\right\} \subset\left\{\tau_{U}^{0}<\zeta^{0}\right\} \quad \mathbf{P}_{x^{-}} \text {a.s. for every } x \in E_{0} .
$$

Here $\tau_{U}^{0}:=\inf \left\{t>0: X_{t}^{0} \notin U\right\}$. This shows that (5.22) almost surely under measure $\mathbf{n}$. Since condition (A.2)" is invariant under the strict time change 
as in the proof of the preceding theorem, condition (A.3) is automatically satisfied. This proves our claim.

Note that condition (5.60) is weaker than the following condition

$$
\mathbf{P}_{x}^{0}\left(\zeta^{0}<\infty\right)=\mathbf{P}_{x}^{0}\left(\zeta^{0}<\infty, X_{\zeta-}^{0}=a\right) \quad \text { for every } x \in E_{0} .
$$

\section{Examples and application}

Several basic examples of Theorem 5.15 have been exhibited in $[16, \S 6]$ when $X^{0}$ are symmetric diffusions on $E_{0}$ in which cases their extensions $X$ are symmetric diffusions on $E$ by [16, Theorem 4.1] there or by Theorem $5.15(\mathrm{v})$ of the present paper. In this section, we first consider a simple case where $X^{0}$ is of pure jump type and admits no killings inside $E_{0}$. A typical example of such a process is a censored stable process on an Euclidean open set studied in [3]. We then consider the case that $X^{0}$ is an absorbing barrier non-symmetric diffusion on an Euclidean domain. As an application, we finally consider an extension of $X^{0}$ by reflecting at infinitely many holes (obstacles).

\subsection{Extending censored stable processes in Euclidean domains}

Let $D$ be an open $n$-set in $\mathbb{R}^{n}$, that is, there exists a constant $C_{1}>0$ such that

$$
m(B(x, r)) \geq C_{1} r^{n} \quad \text { for all } x \in D \text { and } 0<r \leq 1 .
$$

Here $m$ is the Lebesgue measure on $\mathbb{R}^{n}, B(x, r):=\left\{y \in \mathbb{R}^{n}:|x-y|<r\right\}$ and $|\cdot|$ is the Euclidean metric in $\mathbb{R}^{n}$. Note that bounded Lipschitz domains in $\mathbb{R}^{n}$ are open $n$-set and any open $n$-set with a closed subset having zero Lebesgue measure removed is still an $n$-set. For an $n$-set $D$ (which can be disconnected), consider for $0<\alpha<2$ the Dirichlet space defined by

$$
\begin{aligned}
\mathcal{F} & =\left\{u \in L^{2}(D ; d x): \int_{D \times D} \frac{(u(x)-u(y))^{2}}{|x-y|^{n+\alpha}} d x d y<\infty\right\}, \\
\mathcal{E}(u, v) & =\mathcal{A}_{n, \alpha} \int_{D \times D} \frac{(u(x)-u(y))(v(x)-v(y))}{|x-y|^{n+\alpha}} d x d y, \quad u, v \in \mathcal{F},
\end{aligned}
$$

with $\mathcal{A}_{n, \alpha}=\frac{\alpha 2^{\alpha-1} \Gamma\left(\frac{\alpha+n}{2}\right)}{\pi^{n / 2} \Gamma\left(1-\frac{\alpha}{2}\right)}$. When $D=\mathbb{R}^{n},(\mathcal{E}, \mathcal{F})$ is just the Dirichlet form on $L^{2}\left(\mathbb{R}^{n}, d x\right)$ of the symmetric $\alpha$-stable process on $\mathbb{R}^{n}$.

We refer the reader to [3] for the following facts. The bilinear form $(\mathcal{E}, \mathcal{F})$ is a regular irreducible Dirichlet form on $L^{2}\left(\bar{D} ; 1_{D}(x) d x\right)$ and the associated Hunt process $X$ on $\bar{D}$ may be called a reflected $\alpha$-stable process. It is shown in [6] that $X$ has Hölder continuous transition density functions with respect to 
the Lebesgue measure $d x$ on $\bar{D}$ and therefore $X$ can be refined to start from every point in $\bar{D}$.

The process $X^{0}=\left(X_{t}^{0}, \mathbf{P}_{x}^{0}, \zeta^{0}\right)$ obtained from $X$ by killing upon leaving $D$ is called the censored $\alpha$-stable process in $D$, which has been studied in detail in [3]. The process $X^{0}$ is symmetric with respect to the Lebesgue measure and its Dirichlet form on $L^{2}(D, d x)$ is given by $\left(\mathcal{E}, \mathcal{F}^{0}\right)$, where $\mathcal{F}^{0}$ is the closure of $C_{0}^{1}(D)$ in $\mathcal{F}$ with respect to $\mathcal{E}_{1}:=\mathcal{E}+(\cdot, \cdot)_{L^{2}(D, d x)}$. The process $X^{0}$ has no killings inside $D$ in the sense that

$$
\mathbf{P}_{x}\left(\zeta^{0}<\infty \text { and } X_{\zeta^{0}-}^{0} \in D\right)=0 \quad \text { for every } x \in D .
$$

Let $\tau_{D}:=\inf \left\{t>0: X_{t} \notin D\right\}$. Note that for $\left.\beta>0, u_{\beta}(x)=\mathbf{E}_{x}\left[e^{-\beta \tau_{D}}\right)\right]$ is a $\beta$-harmonic function of $X^{0}$ and so it is continuous on $D$ (see $[3,(3.8)]$ ). For any bounded measurable function $f$ on $D$, we extend its definition of $\bar{D}$ by defining $f(x)=0$ on $\partial D$. By [6], $G_{\alpha} f(x):=\mathbf{E}_{x}\left[\int_{0}^{\infty} e^{-\beta t} f\left(X_{t}\right) d t\right]$ is a continuous function on $\bar{D}$. Applying strong Markov property of $X$ at its first exit time $\tau_{D}$ from $D$, we have for $G_{\beta}^{0} f(x):=\mathbf{E}_{x}\left[\int_{0}^{\tau_{D}} e^{-\beta t} f\left(X_{t}\right) d t\right]$,

$$
G_{\beta}^{0} f(x)=G_{\beta} f(x)-\mathbf{E}_{x}\left[e^{-\beta \tau_{D}} G_{\beta} f\left(X_{\tau_{D}}\right)\right] \quad \text { for } x \in D .
$$

Since $x \mapsto \mathbf{E}_{x}\left[e^{-\beta \tau_{D}} G_{\beta} f\left(X_{\tau_{D}}\right)\right]$ is a $\beta$-harmonic function of $X^{0}$ and thus it is continuous on $D$, we conclude that $G_{\beta}^{0} f$ is continuous on $D$. Hence the conditions (A.1) and (A.4)' in $\S 5$ are always satisfied for censored $\alpha$-stable process in any open $n$-set $D$. In view of $[15, \S 5.3]$, a Lévy system of $X^{0}$ is given by $(N(x, d y), d t)$ with

$$
N(x, d y)=2 \mathcal{A}_{n, \alpha}|x-y|^{-(n+\alpha)} d y
$$

and the condition (A.6) of $\S 5$ is clearly satisfied.

Note that if $D_{1}$ is an open subset of $D$, then $X$ and its subprocess killed upon leaving $D_{1}$ have the same class of $m$-polar sets in $D_{1}$. If a closed set $\Gamma \subset \partial D$ has a locally finite and strictly positive $d$-dimensional Hausdorff measure when $n \geq 2$ and is non-empty when $n=1$, then by [3, Theorem 2.5 and Remark 2.2(i)]

$$
\varphi_{\Gamma}(x):=\mathbf{P}_{x}^{0}\left(\zeta^{0}<\infty, X_{\zeta^{0}-}^{0} \in \Gamma\right)>0 \quad \text { for every } x \in D
$$

if and only if $\alpha>n-d$ when $n \geq 2$ and $\alpha>1$ when $n=1$.

In the following $D \subset \mathbb{R}^{n}$ is a proper open $n$-set, $\Gamma$ is a closed subset of $\partial D$ that satisfies the Hausdorff dimensional condition proceeding (6.1). The topology on $D^{*}=D \cup\{a\}$ will be defined in the following three special cases separately.

(i) $D$ is an open $n$-set, $\Gamma=\partial D$, and $\alpha \in(n-d, n)$. Let $D^{*}$ be the one point compactification of $D$. Note that $\varphi(x)=1$ on $D$ with $D$ is bounded, and $0<\phi<1$ on $D$ when $D$ is unbounded with compact boundary. 
(ii) $D$ is an $n$-open set having disconnected boundary $\partial D$. A prototype is a bounded domain $D$ with one or several holes in its interior. Suppose that $\partial D=\Gamma \cup \Gamma_{2}$, where $\Gamma$ and $\Gamma_{2}$ are non-trivial disjoint open subsets of $\partial D$, with $\Gamma$ being compact and satisfying the Hausdorff dimensional condition proceeding (6.1) and $\alpha \in(n-d, n)$. In this case, $0<\varphi_{\Gamma}(x) \leq 1$ for $x \in D$. We prescribe a topology on $D^{*}$ as follows. A subset $U \subset D^{*}$ containing the point $\{a\}$ is a neighborhood of $a$ if there is an open set $U_{1} \subset \mathbb{R}^{d}$ containing $\Gamma_{1}$ such that $U_{1} \cap D=U \backslash\{a\}$. In other words, $D^{*}=D \cup\{a\}$ is obtained from $D$ by identifying $\Gamma$ into one point $\{a\}$.

(iii) $\alpha>1=n, D=(0, \infty)$ and $\Gamma=\{0\}$. In this case $\varphi_{\Gamma}(x)=1 . D^{*}=$ $[0, \infty)$.

In every case, condition (A.2)' in $\S 5$ is fulfilled. Indeed the first half of (A.2)' follows from (6.1). Its second half can be also verified although the proof will be spelled out elsewhere. Consequently, condition (A.3) is automatically satisfied by Theorem 5.17. Therefore, in each case, we can construct the extension $X$ on $D^{*}$ of $X^{0}$ on $D$ satisfying the properties of Theorem 5.15 by means of the Poisson point process around $\{a\} . X$ is a standard process by Theorem 5.16 but admits no jump from $D$ to $a$ nor from $a$ to $D$.

In case (iii), $X$ coincides with the process on $[0, \infty)$ considered in the beginning of this section and may be called a reflecting $\alpha$-stable process. But it differs from the two closely related processes on $[0, \infty)$ that are defined by the symmetric $\alpha$-stable process $x_{t}$ on $\mathbb{R}$ as

$$
X_{t}^{(1)}=\left\{\begin{array}{cr}
x_{t} & t<\sigma_{0} \\
x_{t}-\inf _{\sigma_{0} \leq s \leq t} x_{s} & t \geq \sigma_{0}
\end{array}, \quad X_{t}^{(2)}=\left|x_{t}\right|,\right.
$$

and investigated in detail by S. Watanabe [32], because both $X^{(1)}$ and $X^{(2)}$ admit jumps from $(0, \infty)$ to 0 .

Note that given an open $n$-set with disconnected boundary, extensions in case (i) and (ii) can be different. For example for $D=\left\{x \in \mathbb{R}^{n}: 1<|x|<2\right\}$ with $\Gamma:=\left\{x \in \mathbb{R}^{n}:|x|=1\right\}$, the process $X$ in case (ii) is transient and gets "birth" only when $X^{0}$ approaches $\Gamma$, while in case (i), the extension process is conservative and gets "birth" when $X^{0}$ approaches $\partial D$.

\subsection{Extending non-symmetric diffusions in Euclidean domains}

Let $D$ be a proper domain in $\mathbb{R}^{n}$ and $m$ be the Lebesgue measure on $D$. Assume that $\partial D$ is regular for Brownian motion, or, equivalently, for $\frac{1}{2} \Delta$. Let

$$
\begin{aligned}
\mathcal{L} & =\frac{1}{2} \nabla \cdot(a \nabla)+b \cdot \nabla \\
& =\frac{1}{2} \sum_{i, j=1}^{n} \frac{\partial}{\partial x_{i}}\left(a_{i j} \frac{\partial}{\partial x_{j}}\right)+\sum_{i=1}^{n} b_{i} \frac{\partial}{\partial x_{i}},
\end{aligned}
$$


where $a: \mathbb{R}^{n} \rightarrow \mathbb{R}^{d} \otimes \mathbb{R}^{n}$ is a measurable, symmetric $(n \times n)$-matrix-valued function which satisfies the uniform elliptic condition

$$
\lambda^{-1} I_{n \times n} \leq a(\cdot) \leq \lambda I_{n \times n}
$$

for some $\lambda \geq 1$ and $b=\left(b_{1}, \cdots, b_{n}\right): \mathbb{R}^{n} \rightarrow \mathbb{R}^{n}$ are measurable functions which could be singular such that

$$
1_{D}|b|^{2} \in \mathbf{K}\left(\mathbb{R}^{n}\right), \quad \sum_{i=1}^{n} \frac{\partial b_{i}}{\partial x_{i}}=0 \text { on } D .
$$

Here $\mathbf{K}\left(\mathbb{R}^{n}\right)$ denote the Kato class functions on $\mathbb{R}^{n}$. We refer the reader to [7] for its definition. We only mention here that $L^{p}\left(\mathbb{R}^{n}, d x\right) \subset \mathbf{K}\left(\mathbb{R}^{n}\right)$ for $p>n / 2$.

Let $X^{0}$ be the diffusion in $D$ with infinitesimal generator $\mathcal{L}$ with Dirichlet boundary condition on $\partial D$. It is clearly that $X^{0}$ has a weak dual diffusion $\widehat{X}^{0}$ in $D$ with respect to the Lebesgue measure $m$ on $D$ whose generator is $\mathcal{L}^{*}$, the dual operator of $\mathcal{L}$ with Dirichlet boundary condition on $\partial D$ so that $X^{0}$ satisfies condition (A.1). The conditions (A.4)', (A.5) are satisfied by [7, Lemma 5.7 and Theorem 5.11]. Condition (A.2)' is also satisfied. Its first half is clear and the proof of the second half will be spelled out elsewhere. So condition (A.3) is automatically satisfied by Theorem 5.17 and we can apply Theorem 5.15 to construct a weak duality preserving diffusion extension $X$ of $X^{0}$ to $D^{*}:=D \cup\{a\}$, where the topology on $D^{*}$ can be prescribed as in the three special cases (i)-(iii) in $\S 6.1$.

\subsection{Extending by reflection at infinitely many holes}

In this paper, we restrict ourself to consider duality preserving one-point extension of standard processes $X^{0}$ and $\widehat{X}^{0}$. The method of this paper allows us to do finite many points $\left\{a_{1}, \cdots, a_{n}\right\}$ or countably infinite many points $\left\{a_{1}, \cdots, a_{n}, \cdots\right\}$ extensions, with an obviously modified conditions on $a_{j}$ 's and with no killings at nor direct jumps between $\left\{a_{1}, a_{2}, \cdots\right\}$, provided that $X^{0}$ is symmetric (that is, $X^{0}=\widehat{X}^{0}$ ). One way to do it is to do one-point extension one at a time. We leave the details to the interested reader.

Thus, for example, consider a domain $D \subset \mathbb{R}^{n}$ whose complement $\mathbb{R}^{n} \backslash D$ consists of a countable number of strictly disjoint, non-accumulating compact holes $\left\{K_{1}, K_{2}, \cdots\right\}$. Let $D^{*}:=D \cup\left\{a_{1}, a_{2}, \cdots\right\}$ be the topological space obtained by shrinking each set $K_{i}$ to a point $a_{i}$ and adding all of them to $D$. Let $D_{0}^{*}=D$ and for each $i \geq 1$, we define $D_{i}^{*}:=D_{i-1}^{*} \cup\left\{a_{i}\right\}$, the space obtained by adding $K_{i}$ to $D_{i-1}^{*}$ as one point just as in (ii) of $\S 6.1$. Given an appropriate symmetric Markov process $X^{0}$ on $D$, for $i \geq 1$, the extension $X^{i}$ to $D_{i}^{*}$ can be constructed from $X^{i-1}$ on $D_{i-1}^{*}$ by means of Theorem 5.15 with $\delta_{0}=0$. The extension $X$ of $X^{0}$ on $D$ to $D^{*}:=D \cup\left\{a_{1}, a_{2}, \cdots\right\}$ is obtained as the limit of $X^{i}$ s. The process $X$ is then symmetric on $D^{*}$ and its Dirichlet form may be described in terms of the Feller measure for $X^{0}$ on $D$ studied in detail in [13], [25] and [4]. 


\section{References}

1. R. M. Blumenthal, Excursions of Markov Processes. Birkhäuser, 1992

2. R. M. Blumenthal and R. K. Getoor, Markov Processes and Potential Theory, Academic Press, 1968

3. K. Bogdan, K. Burdzy and Z.-Q. Chen, Censored stable processes, Probab. Theory Relat. Fields 127 (2003), 89-152

4. Z.-Q. Chen, M. Fukushima and J. Ying, Traces of symmetric Markov processes and their characterizations. Ann. Probab. 34 (2006), 1052-1102

5. Z.-Q. Chen, M. Fukushima and J. Ying, Entrance law, exit system and Lévy system of time changed processes. to appear in Illinois J. Math 50 (2006)

6. Z.-Q. Chen and T. Kumagai, Heat kernel estimates for stable-like processes on d-sets. Stochastic Process Appl. 108 (2003), 27-62

7. Z.-Q. Chen and Z. Zhao, Diffusion processes and second order elliptic operators with singular coefficients for lower order terms. Math. Ann. 302 (1995), 323-357

8. C. Dellacherie, B. Maisonneuve et P. A. Meyer, Probabilités et potentiel, Chap. XVII-XXIV, Hermann, Paris, 1992

9. W. Feller, On boundaries and lateral conditions for the Kolmogorov differential equations. Ann. Math. 65 (1957), 527-570

10. P. J. Fitzsimmons and R. K. Getoor, Revuz measures and time changes. Math. Z. 199 (1988), 233-256

11. P. J. Fitzsimmons and R. K. Getoor, Smooth measures and continuous additive functionals of right Markov processes. In Itô's Stochastic Calculus and Probability Theory, Eds. N. Ikeda, S. Watanabe, M. Fukushima and H. Kunita, pp. 31-49, Springer, 1996

12. P. J. Fitzsimmons and R. G. Getoor, Excursion theory revisited. to appear in Illinois J. Math. 50 (2006)

13. M. Fukushima, On boundary conditions for multi-dimensional Brownian motions with symmetric resolvent densities. J. Math. Soc. Japan 21 (1969), 58-93

14. M. Fukushima, P. He and J. Ying, Time changes of symmetric diffusions and Feller measures, Ann. Probab. 32 (2004), 3138-3166

15. M. Fukushima, Y. Oshima and M. Takeda, Dirichlet Forms and Symmetric Markov Processes. Walter de Gruyter, Berlin, 1994

16. M. Fukushima and H. Tanaka, Poisson point processes attached to symmetric diffusions. Ann. Inst. H. Poincaré Probab. Statist. 41 (2005), 419-459

17. R. K.Getoor, Markov processes: Ray processes and right processes, Lect. Notes in Math. 440, Springer, 1970

18. R. K.Getoor, Duality of Lévy system. Z. Wahrsch. verw. Gebiete 19 (1971), $257-270$

19. R. K. Getoor, Excursions of a Markov process. Ann. Probab. 7 (1979), 244-266

20. R. K. Getoor, Excessive Measures. Birkhäuser, 1990

21. R. K. Getoor and M. J. Sharpe, Naturality, standardness, and weak duality for Markov processes. Z. Wahrsch. verw. Gebiete 67(1984), 1-62

22. P. Hsu: On excursions of reflecting Brownian motion. Trans. Amer. Math. Soc. 296 (1986), 239-264.

23. N. Ikeda and S. Watanabe, Stochastic Differential Equations and Diffusion Processes, Second Edition, North-Holland/Kodansha, 1989

24. K. Itô, Poisson point processes attached to Markov processes, in: Proc. Sixth Berkeley Symp. Math. Stat. Probab. III, 1970, 225-239 
25. H. Kunita, General boundary conditions for multi-dimensional diffusion processes, J. Math. Kyoto Univ.10(1970), 273-335

26. B. Maisonneuve, Exit systems. Ann. Probab. 3 (1975), 399-411.

27. P. A. Meyer, Processus de Poisson ponctuels, d'aprés K. Itô, Séminaire de Probab. V, in: Lecture Notes in Math., Vol,191, Springer, Berlin, 1971, pp. 177190

28. L. C. G. Rogers, Itô excursion theory via resolvents, Z. Wahrsch. Verw. Gebiete 63 (1983), 237-255

29. T. S. Salisbury, On the Itô excursion process, Probab. Theor. Relat. Fields 73 (1986), 319-350.

30. T. S. Salisbury, Construction of right processes from excursions, Probab. Theor. Relat. Fields 73 (1986), 351-367

31. M. J. Sharpe, General Theory of Markov Processes. Academic press, 1988

32. S. Watanabe, On stable processes with boundary conditions, J. Math. Soc. Japan 14(1962), 170-198 